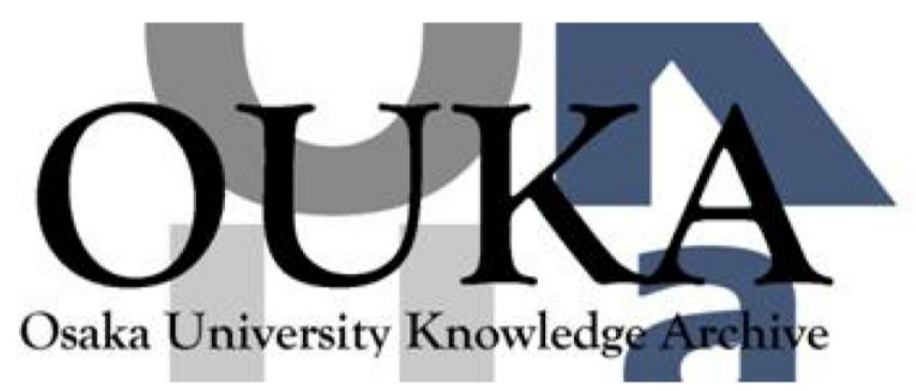

\begin{tabular}{|c|c|}
\hline Title & $\begin{array}{l}\text { A study of mirror symmetry through log mixed } \\
\text { Hodge theory }\end{array}$ \\
\hline Author (s) & Usui, Sampei \\
\hline Citation & $\begin{array}{l}\text { Hodge theory, complex geometry, and } \\
\text { representation theory : NSF-CBMS Regional } \\
\text { Conference in Mathematics Hodge Theory, Complex } \\
\text { Geometry, and Representation Theory, Texas } \\
\text { Christian University, Fort Worth, Texas, June } \\
\text { 18, 2012. p. 285-p.311 }\end{array}$ \\
\hline Issue Date & 2014 \\
\hline oaire:version & AM \\
\hline URL & https://hdl. handle. net/11094/73429 \\
\hline rights & $\begin{array}{l}\text { (- } 2014 \text { by the American Mathematical Society. } \\
\text { All rights reserved. }\end{array}$ \\
\hline Note & \\
\hline
\end{tabular}

Osaka University Knowledge Archive : OUKA

https://ir. Library. osaka-u. ac. jp/

Osaka University 


\title{
A STUDY OF MIRROR SYMMETRY THROUGH LOG MIXED HODGE THEORY
}

\author{
SAMPEI UsUi ${ }^{1}$ \\ Dedicated to Kazuya Kato and Tadashi Ashikaga on their sixtieth birthday, and \\ Phillip Griffiths from whose works the author started his research
}

\begin{abstract}
We hope to understand the Hodge theoretic aspect of mirror symmetry in the framework of the fundamental diagram of log mixed Hodge theory. This paper is a first step. We give a formulation of mirror theorem for quintic threefold and its mirror as the coincidence of log period maps with specified sections under the mirror map ((4) in Introduction). Since a variation of Hodge structure with unipotent monodromy on a punctured disc uniquely extends over the puncture to a log Hodge structure, we can work on and over the boundary point and we can observe clearly in high-resolution the behavior of $\mathbf{Z}$-structure over the boundary point (3.5, (4A) and (4B) in 3.6, 3.7). This is an advantage of log Hodge theory.
\end{abstract}

\section{Contents}

0. Introduction

1. Log mixed Hodge theory

1.1. Category $\mathcal{B}(\log )$

1.2. Ringed space $\left(S^{\log }, \mathcal{O}_{S}^{\log }\right)$

1.3. Gradedly polarized log mixed Hodge structure

1.4. Nilpotent orbit and period map

1.5. Toric variety

1.6. Moduli of log mixed Hodge structures of specified type

1.7. Infinitesimal calculus

Appendix to Section 1. Typographical correction of [KU09, 2.3.2]

2. Examples

2.1. Elliptic curve

2.2. Tate type

2.3. Mirror quintic

2000 Mathematics Subject Classification. Primary 14C30; Secondary 14D07, 32G20.

${ }^{1}$ Partially supported by JSPS. KAKENHI (B) No. 23340008. 


\subsection{Néron model}

3. Quintic threefold

3.1. Picard-Fuchs ideal

3.2. Quintic threefold and its mirror

3.3. Picard-Fuchs equation on B-model of mirror $V^{\circ}$

3.4. A-model of quintic $V$

3.5. Z-structure

3.6. Correspondence table

3.7. Example of computation

3.8. On equivalent statements (1)-(3) in Introduction

3.9. Proof of (4) in Introduction

3.10. Comments

References

\section{Introduction}

Mirror symmetry for A-model of quintic 3-fold $V$ and B-model of its mirror $V^{\circ}$ was predicted in [CDGP91], and proved in the following formulations (1)-(3), which are equivalent. Every statement is near a large radius point $q_{0}$ of the complexified Kähler moduli $\mathcal{K} \mathcal{M}(V)$ and a maximally unipotent monodromy point $p_{0}$ of the complex moduli $\mathcal{M}\left(V^{\circ}\right)$. Let $t:=y_{1} / y_{0}, u:=t / 2 \pi i$ and $q:=e^{t}=e^{2 \pi i u}$ from 3.3 below and respective ones in 3.4 below.

(1) (Potential. [LLuY97], cf. [CoK99, Theorem 11.1.1]) A-model potential $\Phi_{\mathrm{GW}}^{V}$ for $V$ from 3.4 and B-model potential $\Phi_{\mathrm{GM}}^{V^{\circ}}$ for $V^{\circ}$ from 3.3 coincide:

$$
\Phi_{\mathrm{GW}}^{V}(t)=\Phi_{\mathrm{GM}}^{V^{\circ}}(t) .
$$

(2) (Solutions. [Gi96], [Gi97p], cf. [CoK99, Example 11.2.1.3], [P98]) Let

$$
\begin{aligned}
& J_{\mathcal{V}}=5 H\left(1+t H+\frac{d \Phi}{d t} \frac{H^{2}}{5}+\left(t \frac{d \Phi}{d t}-2 \Phi\right) \frac{H^{3}}{5}\right) \\
& I_{\mathcal{V}}=5 H\left(y_{0}+y_{1} H+y_{2} H^{2}+y_{3} H^{3}\right)
\end{aligned}
$$

be the solutions of A- and B-model of Picard-Fuchs equations around $q_{0}$ and $p_{0}$, respectively. (The $y_{i}$ from 3.3 and $\Phi=\Phi_{\mathrm{GW}}^{V}$ from 3.4.) Then, $J_{\mathcal{V}}=I_{\mathcal{V}} / y_{0}$.

(3) (Variation of Hodge structure. [M97], cf. [CoK99, 8.6.1]) The isomorphism $\left(q_{0} \in\right.$ $\overline{\mathcal{K} \mathcal{M}}(V)) \stackrel{\leftarrow}{\leftarrow}\left(p_{0} \in \overline{\mathcal{M}}\left(V^{\circ}\right)\right)$ of neighborhoods of the compactifications, given by the canonical coordinate $q=\exp (2 \pi i u)$, lifts to an isomorphism

$$
\left(\mathcal{H}^{V}, S, \nabla^{\text {middle }}, \mathcal{H}_{\mathbf{Z}}^{V}, \mathcal{F}, 1,[\mathrm{pt}]\right) \stackrel{\sim}{\leftarrow}\left(\mathcal{H}^{V^{\circ}}, Q, \nabla^{\mathrm{GM}}, \mathcal{H}_{\mathbf{Z}}^{V^{\circ}}, \mathcal{F}, \tilde{\Omega}, g_{0}\right)
$$

over the punctured $\mathcal{K} \mathcal{M}(V) \approx \mathcal{M}\left(V^{\circ}\right)$ of B-variation of polarized Hodge structure with specified sections $\tilde{\Omega}$ and $g_{0}$ for $V^{\circ}$ (from 3.3 and $3.6(2 \mathrm{~B})$ below) and A-variation of polarized Hodge structure with specified sections 1 and [pt] for $V$. 
In this paper, we give another formulation (4) below and prove that (4) is equivalent to $(1)-(3)$.

(4) (Log period map) The isomorphism $\left(q_{0} \in \overline{\mathcal{K} \mathcal{M}}(V)\right) \stackrel{\sim}{\leftarrow}\left(p_{0} \in \overline{\mathcal{M}}\left(V^{\circ}\right)\right)$ of neighborhoods of the compactifications uniquely lifts to an isomorphism of B-log variation of polarized Hodge structure with specified sections $\tilde{\Omega}$ and $g_{0}$ for $V^{\circ}$ and A-log variation of polarized Hodge structure with specified sections 1 and [pt] for $V$, whose restriction over the punctured $\mathcal{K} \mathcal{M}(V) \stackrel{\sim}{\mathcal{M}}\left(V^{\circ}\right)$ coincides with the isomorphism of variations of polarized Hodge structure with specified sections in (3).

This rephrases as follows. Let $\sigma$ be the monodromy cone transformed by a level structure into End of a reference fiber of the local system for A- and B- models. Then, we have a commutative diagram of horizontal log period maps

$$
\begin{gathered}
\left(q_{0} \in \overline{\mathcal{K} \mathcal{M}}(V)\right) \stackrel{\leftarrow}{\leftarrow}\left(p_{0} \in \overline{\mathcal{M}}\left(V^{\circ}\right)\right) \\
\searrow \\
\left(\left[\sigma, \exp \left(\sigma_{\mathbf{C}}\right) F_{0}\right] \in \Gamma(\sigma)^{g p} \backslash D_{\sigma}\right)
\end{gathered}
$$

with extensions of specified sections in $(3)$, where $\left(\sigma, \exp \left(\sigma_{\mathbf{C}}\right) F_{0}\right)$ is the nilpotent orbit and $\Gamma(\sigma)^{\mathrm{gp}} \backslash D_{\sigma}$ is the fine moduli of log Hodge structures of specified type which will be explained in Section 1 below.

Let $D$ be a classifying space of (mixed) Hodge structures of specified type. In the series of joint works [KU99], [KU02] and [KU09] for pure case, [KNU08], [KNU09], [KNU11] and [KNU12] for mixed case, we constructed various kind of extensions of $D$ and showed their relation as the following diagram:

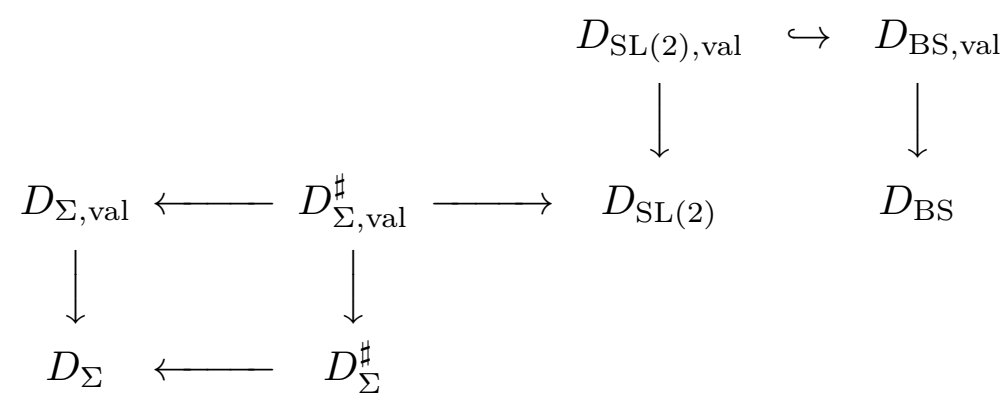

We call this Fundamental Diagram, because this yields a global framework. Indeed, this diagram, taken the quotients by a discrete group $\Gamma$, shows the relation of various kind of compactifications of $\Gamma \backslash D$, i.e., shows the relation of various kind of limits of a series of points of $\Gamma \backslash D$. We hope to observe Hodge theoretic side of mirror symmetry in Fundamental Diagram. The above formulation (4), which is local, is a first step.

In Section 1, we give a brief guide to log mixed Hodge theory from [KNU12]. For the proof of above (4), local theory of log Hodge structure from [KU09] is enough, but we review here the newest results of global theory of mixed version for the communication among wider people in the future. In Section 2, we write some examples of moduli space of log mixed Hodge structures from [KU09] and [KNU12]. In Section 3, we 
review mirror symmetry of quintic 3 -fold and its mirror, then we extend variations of Hodge structures from the formulation (3) over the limit points. Especially, we clarify extension of $\mathbf{Z}$-structures by using log Hodge theory, and prove the formulation (4) is equivalent to the other formulations (1)-(3). We notice that $N$ in Section 3 is $-N$ of $N$ in Sections 1-2.

Acknowledgements. The author would like to thank Kazuya Kato and Chikara Nakayama for series of exciting joint works. He would also like to thank Yukiko Konishi for stimulating discussions. This paper is an answer to the question of Claire Voisin in the conference talk of Phillip Griffiths at Banff 2008. The author is grateful to the referee for recommending to improve Sections 1-2 as a user-friendly introduction.

\section{Log mixed Hodge theory}

This section is a review of some results of log mixed Hodge theory from [KU09] and [KNU12]. We will explain the construction of the fine moduli $\Gamma \backslash D_{\Sigma}$ of log mixed Hodge structures of specified type $\Phi=(\Lambda, \Sigma, \Gamma)$, i.e., the following theorem.

Theorem. Given a data $\Phi$ with $\Gamma$ neat. Then, the functor $\mathrm{LMH}_{\Phi}$ is represented by $\Gamma \backslash D_{\Sigma}$ in the category $\mathcal{B}(\log )$, i.e., $\mathrm{LMH}_{\Phi}(*) \stackrel{\sim}{\rightarrow} \operatorname{Map}\left(*, \Gamma \backslash D_{\Sigma}\right)$ as functors on $\mathcal{B}(\log )$.

This theorem yields a generalization and refinement of period map and also normal function of Griffiths ([Gr68a], [Gr68b]).

We also add results of Néron model from [KNU12] for future use.

\subsection{Category $\mathcal{B}(\log )$}

Let $Z$ be an analytic space and $S$ be a subset of $Z$. The strong topology of $S$ in $Z$ is the strongest one among the topologies on $S$ with respect to which, for any analytic space $A$ and any morphism $f: A \rightarrow Z$ of analytic spaces satisfying $f(A) \subset S$ as sets, $f: A \rightarrow S$ is continuous.

For a local ringed space $S$, a $\log$ structure on $S$ is a sheaf of monoids $M$ on $S$ together with a monoid homomorphisim $\alpha: M \rightarrow \mathcal{O}_{S}$ satisfying $\alpha^{-1} \mathcal{O}_{S}^{\times} \stackrel{\sim}{\rightarrow} \mathcal{O}_{S}^{\times}$. Here $\mathcal{O}_{S}$ is viewed as a sheaf of monoids by multiplication. An fs log structure is a log structure which satisfies the condition "fs", i.e., finitely generated, integral and saturated (for the precise definition, see [KU09, 2.1.5]).

An analytic space is call log smooth if, locally, it is isomorphic to an open set of toric variety. By a log manifold ([KU09, 3.5.7]), we mean a log local ringed space over $\mathbf{C}$ which has an open covering $\left(U_{\lambda}\right)_{\lambda}$ with the following property: For each $\lambda$, there exist a $\log$ smooth fs $\log$ analytic space $Z_{\lambda}$, a finite subset $I_{\lambda}$ of global log differential 1-forms $\Gamma\left(Z_{\lambda}, \omega_{Z_{\lambda}}^{1}\right)$, and an isomorphism of log local ringed spaces over $\mathbf{C}$ between $U_{\lambda}$ and an open subset in the strong topology of

$$
S_{\lambda}:=\left\{z \in Z_{\lambda} \mid \text { the image of } I_{\lambda} \text { in the stlk } \omega_{z}^{1} \text { is zero }\right\}
$$

in $Z_{\lambda}$. This $S_{\lambda}$ becomes an analytically constructible subse of $Z_{\lambda}([\mathrm{KU} 09,3.5 .9])$. 
$E_{\sigma}$ in 1.6 below and $\Gamma \backslash D_{\Sigma}$ in 1.7, for $\Gamma$ being neat and strongly compatible with $\Gamma$, below are log manifolds. "Slits" appear because of the Griffiths tarnsversality condition imposed log point-wisely, i.e., the small Griffiths transversality condition in 1.3 below.

A typical example of slit is as follows:

Example. $S:=((\mathbf{C} \times \mathbf{C}) \backslash(\{0\} \times \mathbf{C})) \cup\{(0,0)\} \subset Z:=\mathbf{C}^{2}$.

\subsection{Ringed space $\left(S^{\log }, \mathcal{O}_{S}^{\log }\right)$}

Let $S$ be an object of $\mathcal{B}(\log )$.

As a set, we define

$$
S^{\log }:=\left\{(s, h) \mid s \in S, h: M_{s}^{\text {gp }} \rightarrow \mathbf{S}^{1} \text { homomorphism s.t. } h(u)=u /|u|\left(u \in \mathcal{O}_{S, s}^{\times}\right)\right\} .
$$

Here $M^{\mathrm{gp}}$ is the sheaf of abelian groups associated to $M$ (i.e., fractions of $M$ ), and $\mathbf{S}^{1}$ is the unit circle in $\mathbf{C}$. We endow $S^{\log }$ with the weakest topology for which the following maps (1) and (2) are continuous.

(1) $\tau: S^{\log } \rightarrow S,(s, h) \mapsto s$.

(2) For any open $U \subset S$ and any $f \in \Gamma\left(U, M^{\mathrm{gp}}\right), \tau^{-1}(U) \rightarrow \mathbf{S}^{1},(s, h) \mapsto h\left(f_{s}\right)$.

Then, $\tau$ is proper and surjective map with fibers $\tau^{-1}(s)=\left(\mathbf{S}^{1}\right)^{r(s)}$, where $r(s):=$ $\operatorname{rank}\left(M^{\mathrm{gp}} / \mathcal{O}_{S}^{\times}\right)_{s}$. Note that $r(s)$ varies with $s \in S$.

We define the sheaf of logarithms $\mathcal{L}$ of $M^{\text {gp }}$ on $S^{\log }$ as the fiber product:

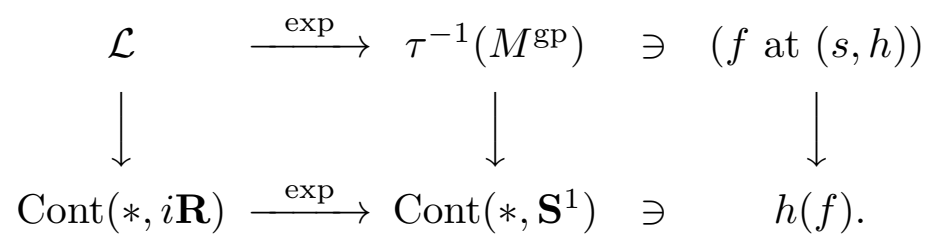

A commutative diagram

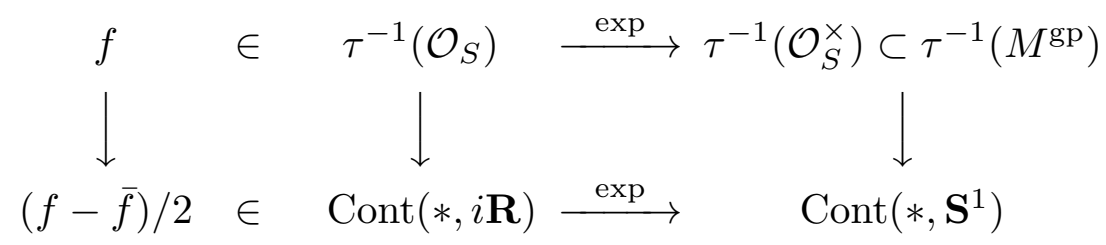

induces a homomorphism $\iota: \tau^{-1}\left(\mathcal{O}_{S}\right) \rightarrow \mathcal{L}$. We define

$$
\mathcal{O}_{S}^{\log }:=\frac{\tau^{-1}\left(\mathcal{O}_{S}\right) \otimes \operatorname{Sym}_{\mathbf{Z}}(\mathcal{L})}{\left(f \otimes 1-1 \otimes \iota(f) \mid f \in \tau^{-1}\left(\mathcal{O}_{S}\right)\right)}
$$

Then, we have a morphism $\tau:\left(S^{\log }, \mathcal{O}_{S}^{\log }\right) \rightarrow\left(S, \mathcal{O}_{S}\right)$ of ringed spaces over $\mathbf{C}$.

For $s \in S$ and $t \in \tau^{-1}(s) \subset S^{\text {log }}$, let $t_{j}(1 \leq j \leq r(s))$ be elements of $\mathcal{L}_{t}$ such that the images in $\left(M^{\mathrm{gp}} / \mathcal{O}_{S}^{\times}\right)_{s}$ of $\exp \left(t_{j}\right)$ form a basis. Then $\mathcal{O}_{S, t}^{\log }$ is a polynomial ring in $r(s)$ variables $t_{j}(1 \leq j \leq r(s))$ over $\mathcal{O}_{S, s}([\mathrm{KU} 09,2.2 .5])$. 


\subsection{Gradedly polarized log mixed Hodge structure}

Let $S \in \mathcal{B}(\log )$.

A pre-gradedly polarized log mixed Hodge structure (pre-GPLMH for short) on $S$ is a quadruple $H=\left(H_{\mathbf{Z}}, W,\left(\langle,\rangle_{w}\right)_{w}, H_{\mathcal{O}}\right)$ consisting of a locally constant sheaf of $\mathbf{Z}$ free modules of finite rank on $S^{\log }$ endowed with an increasing filtration $W$ of $H_{\mathbf{Q}}:=$ $\mathbf{Q} \otimes H_{\mathbf{Z}}$ together with a degenerate $(-1)^{w}$-symmetric $\mathbf{Q}$-bilinear form on each $\operatorname{gr}_{w}^{W}$ and a locally free $\mathcal{O}_{S}$-module on $S$ which satisfy the following conditions. There exists an isomorphism $\mathcal{O}_{S}^{\log } \otimes_{\mathbf{Z}} H_{\mathbf{Z}} \simeq \mathcal{O}_{S}^{\log } \otimes_{\mathcal{O}_{S}} H_{\mathcal{O}}$ (log Riemann-Hilbert correspondence), and there exists a decreasing filtration $F H_{\mathcal{O}}$ of $H_{\mathcal{O}}$ such that $F^{p} H_{\mathcal{O}}$ and $H_{\mathcal{O}} / F^{p} H_{\mathcal{O}}$ are locally free for all $p$. Put $F^{p}:=\mathcal{O}_{S}^{\log } \otimes_{\mathcal{O}_{S}} F^{p} H_{\mathcal{O}}$. Then it can be seen $\tau_{*} F^{p}=F^{p} H_{\mathcal{O}}$. Orthogonality condition $\left\langle F^{p}\left(\operatorname{gr}_{w}^{W}\right), F^{q}\left(\operatorname{gr}_{w}^{W}\right)\right\rangle_{w}=0(p+q>w)$ holds.

A pre-GPLMH on $S$ is a gradedly polarized log mixed Hodge structure (GPLMH for short) on $S$ if its pullback to each $s \in S$ is a GPLMH on $s$ in the following sense. Let $\left(H_{\mathbf{Z}}, W,\left(\langle,\rangle_{w}\right)_{w}, H_{\mathcal{O}}\right)$ be a pre-GPLMH on a log point $s$.

(1) $W$-relative $N$-filtration $M(N, W)$ exists for any logarithm $N$ of local monodromy of the local system $\left(H_{\mathbf{R}}, W,\left(\langle,\rangle_{w}\right)_{w}\right)$ (admissibility).

(2) $\nabla F^{p} \subset \omega_{s}^{1, \log \otimes F^{p-1}}$, where $\omega_{s}^{1, \log }$ is the log differential 1-forms on $\left(s^{\log }, \mathcal{O}_{s}^{\log }\right)$ and $\nabla$ is the $\log$ Gauss-Manin connection defined by $\nabla:=d \otimes 1_{H_{\mathbf{Z}}}: \mathcal{O}_{s}^{\log } \otimes H_{\mathbf{Z}} \rightarrow \omega_{s}^{1, \log } \otimes H_{\mathbf{Z}}$ (Griffiths transversality).

(3) Let $t \in s^{\log }$, and $a: \mathcal{O}_{s, t}^{\log } \rightarrow \mathbf{C}$ be a specialization (i.e., a $\mathbf{C}$-algebra homomorphism). Put $F(a):=\mathbf{C} \otimes_{\mathcal{O}_{s, t}^{\log }} F_{t}$ a filtration on $H_{\mathbf{C}, t}$. Then $\left(H_{\mathbf{Z}, t}\left(\operatorname{gr}_{w}^{W}\right),\langle,\rangle_{w}, F(a)\right)$ is a polarized Hodge structure of weight $w$ in the usual sense for all $w$ and for all sufficiently twisted specialization $a$ in the following sense (positivity).

Fix a subset $\left(q_{j}\right)_{1 \leq j \leq n}$ of the $\log$ structure $M_{s}$ whose image in $M_{s} / \mathcal{O}_{s}^{\times}$generates $M_{s} / \mathcal{O}_{s}^{\times}$as monoid. A specialization $a$ is sufficiently twisted if $\exp \left(a\left(\log q_{j}\right)\right)$ is sufficiently near 0 for all $j$.

Remark. Admissibility condition of local monodromy plays an important role. The above condition (1) is the simplified version in [K86] under the existence of graded polarization. Sometimes we meet more general situation without assuming graded polarization. In this case, admissibility condition is described in [KNU12, §1] as follows.

The local monodromy of $\left(H_{\mathbf{R}}, W\right)$ is admissible if the local monodromy cone $\sigma$ of $\left(H_{\mathbf{R}}, W\right)$ consisting of the monodromy logarithms satisfies the following condition at every point of $S^{\log }$ : For every face $\tau \prec \sigma$, there exists a $W$-relative $\tau$-filtration $M(\tau, W)$ satisfying the following (4)-(6).

(4) If $\tau \prec \sigma$ and $N \in \sigma$, then $N M(\tau, W)_{w} \subset M(\tau, W)_{w}$ for all $w \in \mathbf{Z}$.

(5) If $\tau \prec \sigma$ and $N \in \tau$, then $N M(\tau, W)_{w} \subset M(\tau, W)_{w-2}$ for all $w$.

(6) If $\tau \prec \sigma$ and $N \in \sigma$ and if $\tau^{\prime} \prec \sigma$ is the smallest face containing $\tau$ and $N$, then $M\left(\tau^{\prime}, W\right)=M(N, M(\tau, W))$, where the right-hand side is $M(\tau, W)$-relative $N$ filtration.

When $S$ is log smooth (1.1), a gradedly polarized log mixed Hodge structure $H$ on $S$ is called a log variation of gradedly polarized mixed Hodge structure if it satisfies the 
big Griffiths transversality condition:

$$
\left(d \otimes 1_{H_{\mathbf{C}}}\right) F^{p} \subset \omega_{S}^{1, \log } \otimes F^{p-1} \quad \text { for all } p,
$$

where $d \otimes 1_{H_{\mathbf{C}}}: \mathcal{O}_{S}^{\log } \otimes_{\mathbf{C}} H_{\mathbf{C}} \rightarrow \omega_{S}^{1, \log } \otimes_{\mathbf{C}} H_{\mathbf{C}}$.

Let $S$ be as above. The condition (7) is stronger than the condition (2) for each log point $s \in S$ endowed with the pullback log structure. In contrast with the former, we call the latter the small Griffiths transversality (cf. [KU09, 2.4.9] for pure case).

\subsection{Nilpotent orbit and period map}

Fix a data $\Lambda:=\left(H_{0}, W,\left(\langle,\rangle_{w}\right)_{w},\left(h^{p, q}\right)_{p, q}\right)$, where $H_{0}$ is a free $\mathbf{Z}$-module of finite rank, $W$ is an increasing filtration on $H_{0, \mathbf{Q}}:=\mathbf{Q} \otimes H_{0},\langle,\rangle_{w}$ is a non-degenerate $(-1)^{w}$-symmetric bilinear form on $\operatorname{gr}_{w}^{W}$, and $\left(h^{p, q}\right)_{p, q}$ is a set of Hodge numbers. Let $D$ be the classifying space of gradedly polarized mixed Hodge structures for the data $\Lambda$, and let $\check{D}$ be the "compact dual" ([U84], [KNU12]). For $A=\mathbf{Z}, \mathbf{Q}, \mathbf{R}, \mathbf{C}$, let $G_{A}:=$ $\operatorname{Aut}\left(H_{0, A}, W,\left(\langle,\rangle_{w}\right)_{w}\right)$, and, for $A=\mathbf{Q}, \mathbf{R}, \mathbf{C}$, let $\mathfrak{g}_{A}:=\operatorname{End}\left(H_{0, A}, W,\left(\langle,\rangle_{w}\right)_{w}\right)$.

Let $\sigma$ be a nilpotent cone in $\mathfrak{g}_{\mathbf{R}}$, i.e., a cone in $\mathfrak{g}_{\mathbf{R}}$ not containing a line and generated over $\mathbf{R}_{\geq 0}$ by a finite number of mutually commutative nilpotent elements of $\mathfrak{g}_{\mathbf{R}}$. A subset $Z \subset \check{D}$ is a $\sigma$-nilpotent orbit if the following four conditions are satisfied for $F \in Z$.

(1) $Z=\exp \left(\sigma_{\mathbf{C}}\right) F$.

(2) $\sigma$ is admissible with respect to $W$, i.e., there exists $W$-relative $N$-filtration $M(N, W)$ for any $N \in \sigma$.

(3) $N F^{p} \subset F^{p-1}$ for any $N \in \sigma$ any $p$.

(4) If $N, \ldots, N_{n}$ generate $\sigma$, then $\exp \left(\sum_{j} i y_{j} N_{j}\right) F \in D$ for sufficiently large $y_{j}(\forall j)$.

In this situation, we say that $(\sigma, Z)$ is a nilpotent orbit. We say also that $(\sigma, F)$ or even $\left(N, \ldots, N_{n}, F\right)$ generates a nilpotent orbit.

A weak fan $\Sigma$ in $\mathfrak{g}_{\mathbf{Q}}$ is a set of nilpotent cones in $\mathfrak{g}_{\mathbf{R}}$, defined over $\mathbf{Q}$, such that

(5) Every $\sigma \in \Sigma$ is admissible.

(6) If $\sigma \in \Sigma$ and $\tau \prec \sigma$, then $\tau \in \Sigma$.

(7) If $\sigma, \sigma^{\prime} \in \Sigma$ have a common interior point and if there exists $F \in \check{D}$ such that $(\sigma, F)$ and $\left(\sigma^{\prime}, F\right)$ generate nilpotent orbits, then $\sigma=\sigma^{\prime}$.

Note that this notion is wider than the familiar notion of fan. Weak fan is necessary to construct even a connected Néron model (2.4 below). See [KNU12, 7.2] for an example which shows that fan is too narrow and weak fan is necessary.

Let $\Sigma$ be a weak fan and $\Gamma$ be a subgroup of $G_{\mathbf{Z}} . \Sigma$ and $\Gamma$ are strongly compatible if the following (8) and (9) hold:

(8) If $\sigma \in \Sigma$ and $\gamma \in \Gamma$, then $\operatorname{Ad}(\gamma) \sigma \in \Sigma$.

(9) For any $\sigma \in \Sigma, \sigma$ is generated over $\mathbf{R}_{\geq 0}$ by $\log \Gamma(\sigma)$, where $\Gamma(\sigma):=\Gamma \cap \exp (\sigma)$. 
Using the argument in [KU09, Ch. 2], we will briefly explain that the notion of a gradedly polarized log mixed Hodge structure on an fs log point is equivalent to the notion of a nilpotent orbit in mixed case. The basic idea is that, on the ringed space $\left(S^{\log }, \mathcal{O}_{S}^{\log }\right)(S \in \mathcal{B}(\log ))$, any local system with unipotent monodromy becomes isomorphic to the constant sheaf after shrinking $S$ and tensoring $\mathcal{O}_{S}^{\text {log }}$ (Proposition 2.3.2 loc.cit., see Appendix to Section 1 below).

Let $S \in \mathcal{B}(\log )$. Let $H=\left(H_{\mathbf{Z}}, W,\left(\langle,\rangle_{w}\right)_{w}, H_{\mathcal{O}}\right)$ be a pre-gradedly polarized log mixed Hodge structure on $S$ of Hodge type $\left(h^{p, q}\right)_{p, q}$. Fix a base point $t_{0} \in S^{\log }$ and let $H_{0}=H_{\mathbf{Z}, t_{0}}$, the stalk of $H_{\mathbf{Z}}$ at $t_{0}$, endowed with the induced $W$ and $\left(\langle,\rangle_{w}\right)_{w}$. Let $\Gamma$ be a subgroup of $G_{\mathbf{Z}}$ containing the monodromy group of $\left(H_{\mathbf{Z}}, W,\left(\langle,\rangle_{w}\right)_{w}\right)$. Let $\mu$ be a $\Gamma$ level structure, i.e., a section of the sheaf $\Gamma \backslash \mathcal{I} \operatorname{som}\left(\left(H_{\mathbf{Z}}, W,\left(\langle,\rangle_{w}\right)_{w}\right),\left(H_{0}, W,\left(\langle,\rangle_{w}\right)_{w}\right)\right)$ on $S^{\log }$. We can consider the set-theoretic period map associated to $H$, analogously to Section 2.5.3 in [KU09].

Let

$$
\check{D}_{\text {orb }}:=\left\{(\sigma, Z) \mid \sigma \text { is a nilpotent cone in } \mathfrak{g}_{\mathbf{R}}, Z=\exp \left(\sigma_{\mathbf{C}}\right) F \text { for some } F \in Z\right\} \text {. }
$$

Define a map

$$
\check{\varphi}: S \rightarrow \Gamma \backslash \check{D}_{\text {orb }}
$$

as follows. Let $s \in S$, and $t \in S^{\log }$ lying over $s$, and let $\tilde{\mu}_{t}:\left(H_{\mathbf{Z}, t}, W,\left(\langle,\rangle_{w}\right)_{w}\right) \stackrel{\sim}{\longrightarrow}$ $\left(H_{0}, W,\left(\langle,\rangle_{w}\right)_{w}\right)$ be a lifting of the germ $\mu_{t}$ of $\mu$ at $t$. Then, via $\tilde{\mu}_{t}$, the action of $\pi_{1}\left(s^{\log }\right)$ on $H_{\mathbf{Z}, t}$ defines a nilpotent action on $H_{0}$ preserving $\left(W,\left(\langle,\rangle_{w}\right)_{w}\right)$. Let $\sigma$ be the cone in $\mathfrak{g}_{\mathbf{R}}$ generated by the logarithms of the actions of elements of $\pi_{1}^{+}\left(s^{\log }\right)$ on $H_{0}$ via $\tilde{\mu}_{t}$. Take generators $N_{j}(1 \leq j \leq n)$ of the cone $\sigma$, and let $h_{j} \in \operatorname{Hom}\left(M_{s} / \mathcal{O}_{s}^{\times}, \mathbf{N}\right) \simeq \pi_{1}^{+}\left(s^{\log }\right)$ be the element corresponding to $\exp N_{j}$. Take elements $q_{k} \in M_{s} \backslash \mathcal{O}_{s}^{\times}(1 \leq k \leq m)$ such that their images in $M_{s} / \mathcal{O}_{s}^{\times}$generate this monoid. Let $a_{0}, a \in \operatorname{sp}(t)$ be specializations, and take $z_{j} \in \mathbf{C}(1 \leq j \leq n)$ such that

$$
\frac{1}{2 \pi i}\left(a\left(\log q_{k}\right)-a_{0}\left(\log q_{k}\right)\right)=\sum_{j=1}^{n} z_{j} h_{j}\left(q_{k}\right) \quad(1 \leq k \leq m) .
$$

Then, by Proposition 2.5.1 loc.cit., we have

$$
F(a)=\exp \left(\sum_{j=1}^{n} z_{j} N_{j}\right) F\left(a_{0}\right)
$$

Hence $Z:=\left\{\tilde{\mu}_{t}(F(a)) \mid a \in \operatorname{sp}(t)\right\}=\exp \left(\sigma_{\mathbf{C}}\right) F\left(a_{0}\right)$ in $\check{D}$. We define

$$
\check{\varphi}(s):=((\sigma, Z) \bmod \Gamma) \in \Gamma \backslash \check{D}_{\text {orb }}
$$

The point $\check{\varphi}(s)$ is independent of the choices of $t$ and $\tilde{\mu}_{t}$. If $S^{\prime} \rightarrow S$ in $\mathcal{B}(\log )$ is strict (i.e., the log structure of $S^{\prime}$ coincides with the pullback of that of $S$ ), then by using the 
pullback of $H$ to $S^{\prime}$ we have a commutative diagram

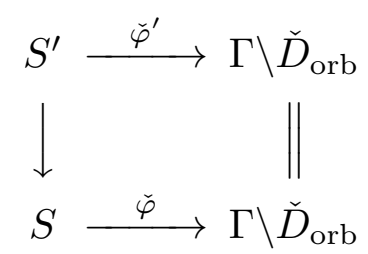

(Lemma 2.5.4 loc.cit.).

Positivity conditions 1.3 (3) and 1.4 (4) coincide (Proposition 2.5.5 (ii) loc.cit.).

Small Griffiths transversality conditions 1.3 (2) and 1.4 (3) coincide, because the composition $H^{0}\left(s^{\log }, \mathcal{O}_{s}^{\log } \otimes H_{\mathbf{Z}}\right) \rightarrow \mathcal{O}_{s}^{\log } \otimes H_{\mathbf{Z}} \stackrel{a}{\rightarrow} H_{\mathbf{C}, t}$ is an isomorphism (Proposition 2.5.5 (i) loc.cit.).

\subsection{Toric variety}

Let $\sigma$ be a nilpotent cone in $\mathfrak{g}_{\mathbf{R}}$ and $\Gamma$ be a subgroup of $G_{\mathbf{Z}}$, and consider the monoid $\Gamma(\sigma)=\Gamma \cap \exp (\sigma)(1.4)$. Assume that the cone generators of $\sigma$ can be taken from $\mathfrak{g}_{\mathbf{Q}}$ and that $\sigma$ is generated over $\mathbf{R}_{\geq 0}$ by $\log \Gamma(\sigma)(1.4(9))$. Let $P(\sigma):=\Gamma(\sigma)^{\vee}=\operatorname{Hom}(\Gamma(\sigma), \mathbf{N})$ be the dual monoid. Define a toric variety and a torus by

$$
\operatorname{toric}_{\sigma}:=\operatorname{Hom}\left(P(\sigma), \mathbf{C}^{\text {mult }}\right) \supset \operatorname{torus}_{\sigma}:=\operatorname{Hom}\left(P(\sigma)^{\mathrm{gp}}, \mathbf{C}^{\times}\right),
$$

where $\mathbf{C}^{\text {mult }}$ is $\mathbf{C}$ regarded as a monoid with multiplication and $P(\sigma)^{\text {gp }}$ is the group generated by the monoid $P(\sigma)$. The exponential sequence $0 \rightarrow \mathbf{Z} \rightarrow \mathbf{C} \rightarrow \mathbf{C}^{\times} \rightarrow 1$ induces the universal covering of the torus

$$
0 \rightarrow \operatorname{Hom}\left(P(\sigma)^{\mathrm{gp}}, \mathbf{Z}\right) \rightarrow \operatorname{Hom}\left(P(\sigma)^{\mathrm{gp}}, \mathbf{C}\right) \stackrel{\mathbf{e}}{\rightarrow} \operatorname{Hom}\left(P(\sigma)^{\mathrm{gp}}, \mathbf{C}^{\times}\right) \rightarrow 1,
$$

where $\operatorname{Hom}\left(P(\sigma)^{\mathrm{gp}}, \mathbf{Z}\right)$ is considered as the fundamental group of torus ${ }_{\sigma}$, and $\mathbf{e}(z \otimes$ $\log \gamma):=e^{2 \pi i z} \otimes \gamma\left(z \in \mathbf{C}, \gamma \in \Gamma(\sigma)^{\mathrm{gp}}=\operatorname{Hom}\left(P(\sigma)^{\mathrm{gp}}, \mathbf{Z}\right)\right)$.

A face $\rho$ of $\sigma$ induces a surjective homomorphism $P(\rho) \leftarrow P(\sigma)$ and hence an open immersion toric $_{\rho} \hookrightarrow$ toric $_{\sigma}$. The origin $0_{\rho} \in$ toric $_{\rho}$ is defined to be the monoid homomorphism $P(\rho) \rightarrow \mathbf{C}^{\text {mult }}$ sending 1 to 1 and all the other elements of $P(\rho)$ to 0 . This point $0_{\rho} \in$ toric $_{\rho}$ is viewed as a point of toric $\sigma$ by the above immersion. Then, as a set, we have

$$
\text { toric }_{\sigma}=\left\{\mathbf{e}(z) 0_{\rho} \mid \rho \prec \sigma, z \in \sigma_{\mathbf{C}} /\left(\rho_{\mathbf{C}}+\log \Gamma(\sigma)^{\mathrm{gp}}\right)\right\} .
$$

For $S:=$ toric $_{\sigma}$, polar coordinate $\mathbf{R}_{\geq 0} \times \mathbf{S}^{1} \rightarrow \mathbf{R}_{\geq 0} \cdot \mathbf{S}^{1}=\mathbf{C}$ induces $\tau: S^{\log } \rightarrow S$ as

$$
\begin{aligned}
& \tau: S^{\log }=\operatorname{Hom}\left(P(\sigma), \mathbf{R}_{\geq 0}^{\text {mult }}\right) \times \operatorname{Hom}\left(P(\sigma), \mathbf{S}^{1}\right) \\
&=\left\{\left(\mathbf{e}(i y) 0_{\rho}, \mathbf{e}(x)\right) \mid \rho \prec \sigma, x \in \sigma_{\mathbf{R}} /\left(\rho_{\mathbf{R}}+\log \Gamma(\sigma)^{\mathrm{gp}}\right), y \in \sigma_{\mathbf{R}} / \rho_{\mathbf{R}}\right\} \\
& \rightarrow S=\operatorname{Hom}\left(P(\sigma), \mathbf{C}^{\text {mult }}\right), \\
& \tau\left(\mathbf{e}(i y) 0_{\rho}, \mathbf{e}(x)\right)=\mathbf{e}(x+i y) 0_{\rho} . \\
& 9
\end{aligned}
$$


Since $0 \rightarrow \rho_{\mathbf{R}} / \log \Gamma(\rho)^{\mathrm{gp}} \rightarrow \sigma_{\mathbf{R}} / \log \Gamma(\sigma)^{\mathrm{gp}} \rightarrow \sigma_{\mathbf{R}} /\left(\rho_{\mathbf{R}}+\log \Gamma(\sigma)^{\mathrm{gp}}\right) \rightarrow 0$ is exact, the fiber of $\tau$, as a set, is described as

$$
\tau^{-1}\left(\mathbf{e}(a+i b) 0_{\rho}\right)=\left\{\left(\mathbf{e}(i b) 0_{\rho}, \mathbf{e}(a+x)\right) \mid x \in \rho_{\mathbf{R}} / \log \Gamma(\rho)^{\mathrm{gp}}\right\} \simeq\left(\mathbf{S}^{1}\right)^{r},
$$

where $r=r(\rho):=\operatorname{rank} \rho$ varies with $\rho \prec \sigma$.

Let $H_{\sigma}=\left(H_{\sigma, \mathbf{Z}}, W,\left(\langle,\rangle_{w}\right)_{w}\right)$ be the canonical locally constant sheaf on $S^{\log }$ given by the representation of $\pi_{1}\left(S^{\log }\right)=\Gamma(\sigma)^{\mathrm{gp}} \subset G_{\mathbf{Z}}=\operatorname{Aut}\left(H_{0}, W,\left(\langle,\rangle_{w}\right)_{w}\right)$. We recall $[\mathrm{KU} 09,2.3 .7]$ in the present situation.

In the general setting as in Proposition 2.3.2 loc.cit. (Appendix to Section 1), the isomorphism $\nu$ appears locally on $X$ depending on a local choice of $\left(q_{j}\right)_{j}$. But in the case $S=$ toric $_{\sigma}$, a canonical $\nu$ exists globally on $S$.

Indeed, let $A$ be a subring of $\mathbf{C}$, let $L:=H_{\sigma, A}$ be the locally constant sheaf on $S^{\text {log }}$ of free $A$-modules of finite rank with unipotent local monodromy, and let $L_{1}$ (denoted by $L_{0}$ in 2.3.2 loc.cit.) be the stalk of $L$ at the unit point $1=0_{\{0\}} \in \operatorname{torus}_{\sigma}=$ $\operatorname{Hom}\left(P(\sigma)^{\mathrm{gp}}, \mathbf{C}^{\times}\right)$regarded as a constant sheaf on $S^{\log }$. We identify $L_{1}$ with $H_{0}$ in 1.4. Then there is a unique isomorphism of $\mathcal{O}_{S}^{\log }$-modules

$$
\nu: \mathcal{O}_{S}^{\log } \otimes_{A} L \stackrel{\sim}{\rightarrow} \mathcal{O}_{S}^{\log } \otimes_{A} L_{1}
$$

satisfying the following (1) and (2) for any Z-basis $\left(q_{j}\right)_{1 \leq j \leq n}$ of $P(\sigma)^{\mathrm{gp}}$. Let $\left(\gamma_{j}\right)_{1 \leq j \leq n}$ be the $\mathbf{Z}$-basis of $\pi_{1}\left(\operatorname{torus}_{\sigma}, 1\right)=\operatorname{Hom}\left(P(\sigma)^{\mathrm{gp}}, \mathbf{Z}\right)$ which is dual to $\left(q_{j}\right)_{1 \leq j \leq n}$, and let $N_{j}: L_{1, A^{\prime}} \rightarrow L_{1, A^{\prime}}$ be the logarithm of $\gamma_{j}$, where $A^{\prime}$ is the subring of $\mathbf{C}$ generated by $A$ and $\mathbf{Q}$.

$$
\nu(1 \otimes L)=\xi^{-1}\left(1 \otimes L_{1}\right) \quad \text { with } \xi=\exp \left(\sum_{j=1}^{n}(2 \pi i)^{-1} \log \left(q_{j}\right) \otimes N_{j}\right) .
$$

(2) Let $\log \left(q_{j}\right)_{1,0}$ be the branch in $\mathcal{O}_{S, 1}^{\log }$ of the germ of $\log \left(q_{j}\right)$ at $1 \in$ torus $_{\sigma}$ which has the value 0 at 1 , and let $\xi_{1,0}=\exp \left(\sum_{j=1}^{n}(2 \pi i)^{-1} \log \left(q_{j}\right)_{1,0} \otimes N_{j}\right)$. Then the map $\xi_{1,0} \circ \nu_{1}: 1 \otimes L_{1} \rightarrow 1 \otimes L_{1}$ is the identity map.

The proof is similar to that of 2.3.2 loc.cit. First fix $\left(q_{j}\right)_{1 \leq j \leq n}$. For the locally constant subsheaf $L^{\prime}:=\xi^{-1}\left(1 \otimes L_{1}\right)$ of $\mathcal{O}_{S}^{\log } \otimes L_{1}$, the isomorphism $\xi_{1,0}^{-1}: L_{1} \rightarrow L_{1}^{\prime}$ of stalks preserves the actions of $\pi_{1}\left(S^{\log }, 1\right) \simeq \pi_{1}\left(\right.$ torus $\left._{\sigma}, 1\right)$, and it is extended uniquely to an isomorphism $\nu: L \stackrel{\sim}{\rightarrow} L^{\prime}$ on $S^{\log }$. This induces an isomorphism of $\mathcal{O}_{S}^{\log }$-modules $\nu: \mathcal{O}_{S}^{\log } \otimes_{A} L \stackrel{\sim}{\rightarrow} \mathcal{O}_{S}^{\log } \otimes_{A} L^{\prime}=\mathcal{O}_{S}^{\log } \otimes_{A} L_{1}$. It is easy to check that $\nu$ is independent of the choice of $\left(q_{j}\right)_{1 \leq j \leq n}$.

\subsection{Moduli of log mixed Hodge structures of type $\Phi$}

Let $\Phi=(\Lambda, \Sigma, \Gamma)$, where $\Lambda$ is from $1.4, \Sigma$ is a weak fan and $\Gamma$ is a subgroup of $G_{\mathbf{Z}}$ such that $\Sigma$ and $\Gamma$ are strongly compatible.

Let $\sigma \in \Sigma$. Let $S=$ toric $_{\sigma}$ and let $H_{\sigma}=\left(H_{\sigma, \mathbf{z}}, W,\left(\langle,\rangle_{w}\right)_{w}\right)$ be the canonical locally constant sheaf on $S^{\log }$ from 1.5. The universal pre-GPLMH $H$ on $\check{E}_{\sigma}:=\operatorname{toric}_{\sigma} \times \check{D}$ 
is given by $H_{\sigma}$ together with the isomorphism $\mathcal{O}_{\check{E}_{\sigma}}^{\log } \otimes_{\mathbf{z}} H_{\sigma, \mathbf{Z}}=\mathcal{O}_{\check{E}_{\sigma}}^{\log } \otimes_{\mathcal{O}_{\check{E}_{\sigma}}} H_{\mathcal{O}}$, where $H_{\mathcal{O}}:=\mathcal{O}_{\check{E}_{\sigma}} \otimes H_{0}$ is the free $\mathcal{O}_{\check{E}_{\sigma}}$-module coming from that on $\check{D}$ endowed with the universal Hodge filtration $F$. Define

$$
E_{\sigma}:=\left\{x \in \check{E}_{\sigma} \mid H(x) \text { is a GPLMH }\right\} .
$$

Note that slits appear in $E_{\sigma}$ because of the small Griffiths transversality 1.3 (2), or equivalently $1.4(3)$.

As a set, define

$$
D_{\Sigma}:=\left\{(\sigma, Z) \in \check{D}_{\text {orb }} \mid \text { nilpotent orbit, } \sigma \in \Sigma, Z \subset \check{D}\right\} .
$$

Let $\sigma \in \Sigma$. Assume $\Gamma$ is neat, i.e., for each $\gamma \in \Gamma$, the subgroup of $\mathbf{C}$ generated by all the eigenvalues of $\gamma$ is torsion-free. A structure as an object of $\mathcal{B}(\log )$ on $\Gamma \backslash D_{\Sigma}$ is introduced by the diagram:

$$
\begin{gathered}
E_{\sigma} \stackrel{\text { GPLMH }}{\subset} \check{E}:=\text { toric }_{\sigma} \times \check{D} \\
\downarrow \sigma_{\mathrm{C} \text {-torsor }} \\
\Gamma(\sigma)^{\mathrm{gp}} \backslash D_{\sigma} \\
\quad \downarrow \text { loc. isom. } \\
\Gamma \backslash D_{\Sigma}
\end{gathered}
$$

Here the action of $h \in \sigma_{\mathbf{C}}$ on $\left(\mathbf{e}(a) 0_{\rho}, F\right) \in E_{\sigma}$ is $\left(\mathbf{e}(h+a) 0_{\rho}, \exp (-h) F\right)$, and the projection to the quotient is described as $\left(\mathbf{e}(a) 0_{\rho}, F\right) \mapsto\left(\rho, \exp \left(\rho_{\mathbf{C}}+a\right) F\right)$. By using the fundamental diagram from Introduction, we transported good properties of the Borel-Serre type compactification $\Gamma \backslash D_{\mathrm{BS}}$, such as Hausdorffness, to the $\sigma_{\mathbf{C}}$-action on $E_{\sigma}$ and showed that the projection $E_{\sigma} \rightarrow E_{\sigma} / \sigma_{\mathbf{C}}=\Gamma(\sigma)^{\mathrm{gp}} \backslash D_{\sigma}$ is a $\sigma_{\mathbf{C}^{-}}$-torsor in the category $\mathcal{B}(\log )$. This is the hardest part of the proof (see [KU09], [KNU12).

Let $S \in \mathcal{B}(\log )$. A log mixed Hodge structure of type $\Phi$ (LMH of type $\Phi$ for short)on $S$ is a pre-GPLMH $H=\left(H_{\mathbf{Z}}, W,\left(\langle,\rangle_{w}\right)_{w}, H_{\mathcal{O}}\right)(1.3)$ endowed with a $\Gamma$-level structure

$$
\mu \in H^{0}\left(S^{\log }, \Gamma \backslash \operatorname{Isom}\left(\left(H_{\mathbf{Z}}, W,\left(\langle,\rangle_{w}\right)_{w}\right),\left(H_{0}, W,\left(\langle,\rangle_{w}\right)_{w}\right)\right)\right)
$$

satisfying the following condition: For any $s \in S$, any $t \in \tau^{-1}(s)=s^{\log }$, any representative $\tilde{\mu}_{t}: H_{\mathbf{Z}, t} \stackrel{\sim}{\rightarrow} H_{0}$, and the local monodromy monoid $P_{s}$ of $H_{\mathbf{Z}}$ at $s$, i.e., $P_{s}:=\operatorname{Image}\left(\operatorname{Hom}\left(\left(M_{S} / \mathcal{O}_{S}^{\times}\right)_{s}, \mathbf{N}\right) \hookrightarrow \pi_{1}\left(s^{\log }\right) \rightarrow \operatorname{Aut}\left(H_{\mathbf{Z}, t}\right)\right)$, there exists $\sigma \in \Sigma$ such that $\sigma$ contains $\tilde{\mu}_{t} P_{s} \tilde{\mu}_{t}^{-1}$ and that $\left(\sigma, \tilde{\mu}_{t}\left(\mathbf{C} \otimes_{\mathcal{O}_{S, t}^{\log }} F_{t}\right)\right)$ generates a nilpotent orbit (1.4). (Then, the smallest such $\sigma$ exists.)

As explained in 1.4, a log mixed Hodge structure of type $\Phi$ is a gradedly polarized log mixed Hodge structure in 1.3 (cf. [KNU12, 2.2.2]).

Theorem. (i) $\Gamma \backslash D_{\Sigma} \in \mathcal{B}(\log )$, which is Hausdorff. If $\Gamma$ is neat, $\Gamma \backslash D_{\Sigma}$ is a log manifold.

(ii) On $\mathcal{B}(\log ), \Gamma \backslash D_{\Sigma}$ represents the functor $\mathrm{LMH}_{\Phi}$ of LMH of type $\Phi$. 
Log period map. Let $S \in \mathcal{B}(\log )$. Then we have an isomorphism

$$
\operatorname{LMH}_{\Phi}(S) \stackrel{\sim}{\rightarrow} \operatorname{Map}\left(S, \Gamma \backslash D_{\Sigma}\right), \quad H \mapsto\left(S \ni s \mapsto\left(\sigma, \exp \left(\sigma_{\mathbf{C}}\right) \tilde{\mu}_{t}\left(\mathbf{C} \otimes_{\mathcal{O}_{S, t}^{\log }} F_{t}\right)\right) \bmod \Gamma\right)
$$

which is functorial in $S$.

This is the log period map mentioned at the beginning of Section 1.

\subsection{Infinitesimal calculus}

Let $S \in \mathcal{B}(\log )$ be a $\log$ manifold. Define $\theta_{S}:=\mathcal{H}_{o} m_{\mathcal{O}_{S}}\left(\omega_{S}^{1}, \mathcal{O}_{S}\right)$.

Theorem. Assume $\Gamma$ is neat. Put $S:=\Gamma \backslash D_{\Sigma}$. Then we have a commutative diagram

$$
\begin{array}{cc}
\theta_{S} \stackrel{\sim}{\longrightarrow} \operatorname{End}\left(H_{\mathcal{O}}, W,\left(\langle,\rangle_{w}\right)_{w}\right) / F^{0} \mathcal{E} n d(\cdots) \\
\cup \\
\theta_{S}^{h} \stackrel{\sim}{\longrightarrow} \quad \operatorname{gr}_{F}^{-1} \mathcal{E} n d\left(H_{\mathcal{O}}, W,\left(\langle,\rangle_{w}\right)_{w}\right)
\end{array}
$$

where $\theta_{S}^{h}$ is the horizontal subbundle of $\theta_{S}$.

Idea of Proof. Taking $\tau_{*}$ of $d \otimes 1_{H_{\mathbf{C}}}: \mathcal{O}_{S}^{\log } \otimes H_{\mathbf{C}} \rightarrow \omega_{S}^{1, \log } \otimes H_{\mathbf{C}}$, we have the GaussManin connection in the $\log$ sense $\nabla: H_{\mathcal{O}} \rightarrow \omega_{S}^{1} \otimes H_{\mathcal{O}}$. Define $\theta_{S} \rightarrow \mathcal{E} n d\left(H_{\mathcal{O}}\right)$, by $\delta \mapsto \nabla_{\delta}$.

In the algebraic context, by the analogous argument of [KO68], the above construction of $\nabla$ is globalized to get an algebraic connection. Note that the above $\nabla$ is nothing but the canonical connection in the sense of [D97] [CaK99, (8.50)].

Example. Let $S:=\Delta^{n+t}$ be a polydisc and $f: X \rightarrow S$ be a projective morphisim of complex manifolds such that the restriction of $f$ over $S^{*}:=\left(\Delta^{*}\right)^{n} \times \Delta^{t}$ is smooth. Put $X^{*}:=f^{-1}\left(S^{*}\right)$. Let $A \subset X$ be a normal crossing divisor such that $A \cap X^{*}$ is relative normal crossing over $S^{*}$. Let $h: X^{*} \backslash A \rightarrow S^{*}$ be the restriction of $f$. Assume that the local monodromy of $H_{\mathbf{R}}:=R^{m} h_{*} \mathbf{R}$ over $S^{*}$ is unipotent. Then, the variation of mixed Hodge structure with graded quotients on $H_{\mathbf{Z}}:=R^{m} h_{*} \mathbf{Z}$ over $S^{*}$ canonically extends to a $\log$ mixed Hodge structure $H$ on $S$ of type $\Phi$ with $\log$ structures $M_{S}:=$ (the one corresponding to the divisor $S \backslash S^{*}$ ), $M_{X}:=$ (the one corresponding to $A$ ), a fan $\Sigma:=$ (faces of the local monodromy cone at $0 \in S$ ), and a monodromy group $\Gamma:=$ $\pi_{1}\left(S^{*}\right)=\pi_{1}\left(S^{\log }\right)$. Let $\varphi=\varphi_{H}: S \rightarrow \Gamma \backslash D_{\Sigma}$ be the corresponding period map. We have a commutative diagram

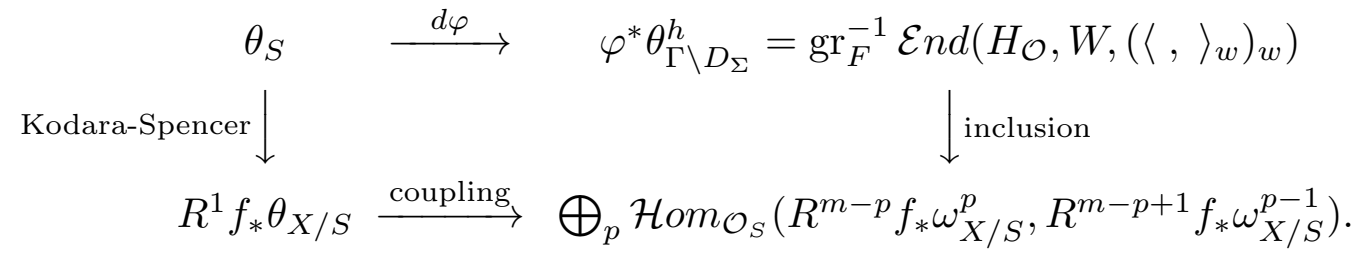

This is a log mixed Hodge theoretic generalization of Griffiths' formula of infinitesimal period map. 


\section{Appendix to Section 1. Typographical correction of [KU09, 2.3.2]}

There are only typographical errors and no other problems in Proposition 2.3.2 of [KU09]. But since they make this fundamental part uncomfortable to read, we add the corrected version here for readers' convenience. The notation and the indications in this appendix are all those loc.cit.

Proposition 2.3.2. Let $X$ be an object of $\overline{\mathcal{A}}_{1}(\log )$ (which contains $\mathcal{B}(\log )$ ), let $A$ be a subring of $\mathbf{C}$, and let $L$ be a locally constant sheaf on $X^{\log }$ of free A-modules of finite rank. Let $x \in X$, let $y$ be a point of $X^{\log }$ lying over $x$, and assume that the local monodromy of $L$ at $y$ is unipotent. Let $\left(q_{j}\right)_{1 \leq j \leq n}$ be a finite family of elements of $M_{X, x}^{\mathrm{gp}}$ whose image in $\left(M_{X}^{\mathrm{gp}} / \mathcal{O}_{X}^{\times}\right)_{x}$ is a $\mathbf{Z}$-basis, and let $\left(\gamma_{j}\right)_{1 \leq j \leq n}$ be the dual $\mathbf{Z}$-basis of $\pi_{1}\left(x^{\log }\right)$ in the duality in 2.2.9. Then if we replace $X$ by some open neighborhood of $x$, we have an isomorphism of $\mathcal{O}_{X}^{\log }$-modules

$$
\nu: \mathcal{O}_{X}^{\log } \otimes_{A} L \stackrel{\sim}{\rightarrow} \mathcal{O}_{X}^{\log } \otimes_{A} L_{0}, \quad L_{0}:=\text { the stalk } L_{y}
$$

where $L_{0}$ is regarded as a constant sheaf, satisfying the condition (1) below. Let $A^{\prime}$ be the subring of $\mathbf{C}$ generated by $A$ and $\mathbf{Q}$, let $L_{0, A^{\prime}}=A^{\prime} \otimes_{A} L_{0}$, and let

$$
N_{j}: L_{0, A^{\prime}} \rightarrow L_{0, A^{\prime}}
$$

be the endmorphism of constant sheaf which is induced by the logarithm of the monodromy action of $\gamma_{j}$ on the stalk $L_{y}$ of the locally constant sheaf L. Lift $q_{j}$ in $\Gamma\left(X, M_{X}^{\mathrm{gp}}\right)$ (by replacing $X$ by an open neighborhood of $x$ ), and let

$$
\xi=\exp \left(\sum_{j=1}^{n}(2 \pi i)^{-1} \log \left(q_{j}\right) \otimes N_{j}\right): \mathcal{O}_{X}^{\log } \otimes_{A^{\prime}} L_{0, A^{\prime}} \stackrel{\sim}{\rightarrow} \mathcal{O}_{X}^{\log } \otimes_{A^{\prime}} L_{0, A^{\prime}} .
$$

Note that the operator $\xi$ depends on the choices of the branches of $\log \left(q_{j}\right)$ in $\mathcal{O}_{X}^{\log }$ locally on $X^{\log }$, but that the subsheaf $\xi^{-1}\left(1 \otimes L_{0}\right)$ of $\mathcal{O}_{X}^{\log } \otimes_{A} L_{0}$ is independent of the choices and hence is defined globally on $X^{\log }$.

(1) The restriction of $\nu$ to $L=1 \otimes L$ induces an isomorphism of locally constant sheaves

$$
\nu: L \stackrel{\sim}{\rightarrow} \xi^{-1}\left(1 \otimes L_{0}\right) .
$$

If we fix branches $\log \left(q_{j}\right)_{y, 0}$ in $\mathcal{O}_{X, y}^{\log }$ of the germs $\log \left(q_{j}\right)_{y}$ at $y(1 \leq j \leq n)$, we can take an isomorphism $\nu$ which satisfies above (1) and also the following (2).

(2) The branch $\xi_{y, 0}$ of the germ $\xi_{y}$, defined by the fixed branches $\log \left(q_{j}\right)_{y, 0}$ of the germs $\log \left(q_{j}\right)_{y}$, satisfies

$$
\nu(1 \otimes v)=\xi_{y, 0}^{-1}(1 \otimes v) \quad \text { for any } v \in L_{y}=L_{0} .
$$

Proof. Let $L^{\prime}$ be the locally constant subsheaf $\xi^{-1}\left(1 \otimes L_{0}\right)$ of $\mathcal{O}_{X}^{\log } \otimes L_{0}$. Fix a branch $\log \left(q_{j}\right)_{y, 0}$ of the germ $\log \left(q_{j}\right)_{y}$ at $y$ for $1 \leq j \leq n$, and let $\nu: L_{y} \rightarrow\left(L^{\prime}\right)_{y}$ be the 
isomorphism of $A$-modules $v \mapsto \xi_{y, 0}^{-1}(1 \otimes v)$, where $\xi_{y, 0}$ is defined by the fixed branches $\log \left(q_{j}\right)_{y, 0}$ of $\log \left(q_{j}\right)_{y}$. Then $\nu$ preserves the local monodromy actions of $\pi_{1}\left(x^{\log }\right)$ on these stalks of the locally constant sheaves $L$ and $L^{\prime}$. In fact, for $v \in L_{0}$ and for $1 \leq k \leq n$,

$$
\begin{aligned}
\gamma_{k}(\nu(v)) & =\gamma_{k}\left(\xi_{y, 0}^{-1}(1 \otimes v)\right)=\gamma_{k}\left(\xi_{y, 0}\right)^{-1} \cdot(1 \otimes v) \\
& =\exp \left(-\left(\sum_{j=1}^{n}\left((2 \pi i)^{-1} \log \left(q_{j}\right)_{y, 0}-\delta_{j k}\right) \otimes N_{j}\right) \cdot(1 \otimes v)\right. \\
& =\xi_{y, 0}^{-1} \exp \left(1 \otimes N_{k}\right)(1 \otimes v)=\xi_{y, 0}^{-1}\left(1 \otimes \gamma_{k}(v)\right)=\nu\left(\gamma_{k}(v)\right)
\end{aligned}
$$

( $\delta_{j k}$ is the Kronecker symbol, and for the signature before $\delta_{j k}$, see Appendix A1). Here the second equality follows from the monodromy action of $\gamma_{k}$ on the locally constant sheaf $L^{\prime}$, the fifth equality follows from the endmorphism $N_{k}$ of the constant sheaf $L_{0}$, and $\gamma_{k}(v)$ in the second last and in the last is the image of the element $v \in L_{0}=L_{y}$ by the monodromy action of $\gamma_{k}$ on the locally constant sheaf $L$ at $y$. Hence there is a unique isomorphism $\nu:\left.\left.L\right|_{x^{\log }} \rightarrow L^{\prime}\right|_{x^{\log }}$ between the pullbacks of $L$ and $L^{\prime}$ to $x^{\log }$ which induces the above isomorphism $\nu$ on the stalks at $y$.

By the proper base change theorem (Appendix A2) applied to the proper map $\tau$ : $X^{\log } \rightarrow X$ and to the sheaf $\mathcal{F}$ of isomorphisms from $L$ to $L^{\prime}$ on $X^{\log }$, the isomorphism $\nu$ extends to an isomorphism $\nu: L \stackrel{\sim}{\rightarrow} L^{\prime}$ if we replace $X$ by some open neighborhood of $x$ in $X$. This isomorphism $\nu$ induces an isomorphism of $\mathcal{O}_{X}^{\log }$-modules

$$
\nu: \mathcal{O}_{X}^{\log } \otimes_{A} L \stackrel{\sim}{\rightarrow} \mathcal{O}_{X}^{\log } \otimes_{A} L^{\prime}=\mathcal{O}_{X}^{\log } \otimes_{A} L_{0}
$$

\section{Examples}

We see some examples of moduli space of log mixed Hodge structures from [KU09] and [KNU12].

\subsection{Elliptic curve ([KU09, Ch. 0])}

Let $H_{0}:=\mathbf{Z}^{2}=\mathbf{Z} e_{1}+\mathbf{Z} e_{2}$, weight $=-1,\langle$,$\rangle anti-symmetric form defined by$ $\left\langle e_{2}, e_{1}\right\rangle=1, D$ the classifying space of polarized Hodge structures of weight -1 , and $\check{D}$ the compact dual. $D$ is identified with upper-half plane $\mathfrak{h}$ via $\tau \leftrightarrow F^{0}:=\mathbf{C}\left(\tau e_{1}+e_{2}\right)$ $(\tau \in \mathfrak{h})$, and $\check{D}=\mathbf{P}^{1}(\mathbf{C})$.

For a fan $\Sigma:=\left\{\mathbf{R}_{\geq 0} N \mid N \in \mathfrak{s l}(2, \mathbf{Q})\right\}$, we have $D_{\Sigma}=D \cup \mathbf{P}^{1}(\mathbf{Q})$. In fact, for $a \in \mathbf{P}^{1}(\mathbf{Q})$, let $V_{a}:=\mathbf{R}\left(a e_{1}+e_{2}\right)$, and define

$$
\begin{aligned}
\sigma_{a} & :=\left\{N \in \mathfrak{s l}(2, \mathbf{R}) \mid N\left(H_{0, \mathbf{R}}\right) \subset V_{a}, N\left(V_{a}\right)=0,\langle x, N x\rangle \geq 0\left(\forall x \in H_{0, \mathbf{R}}\right)\right\}, \\
Z_{a} & :=\left\{F \in \check{D} \mid F^{1} \neq V_{a, \mathbf{C}}\right\} .
\end{aligned}
$$

Then, $\mathbf{P}^{1}(\mathbf{Q}) \ni a \leftrightarrow\left(\sigma_{a}, Z_{a}\right) \in D_{\Sigma} \backslash D$.

For example, $V_{\infty}=\mathbf{R} e_{1}, \sigma:=\sigma_{\infty}=\left(\begin{array}{cc}0 & \mathbf{R}_{\geq 0} \\ 0 & 0\end{array}\right), Z_{\infty}=\mathbf{C} \subset \mathbf{P}^{1}(\mathbf{C})=\check{D}$. Let $\Gamma:=\operatorname{Ker}(\mathrm{SL}(2, \mathbf{Z}) \rightarrow \operatorname{SL}(2, \mathbf{Z} / n \mathbf{Z}))$. It is well-known that $\Gamma$ is neat if and only if $n \geq 3$. 
We assume this. Let $\Gamma(\sigma)^{\mathrm{gp}}:=\Gamma \cap \exp \left(\sigma_{\mathbf{R}}\right)=\left(\begin{array}{cc}1 & n \mathbf{Z} \\ 0 & 1\end{array}\right)$. Then we have a period map and its extension

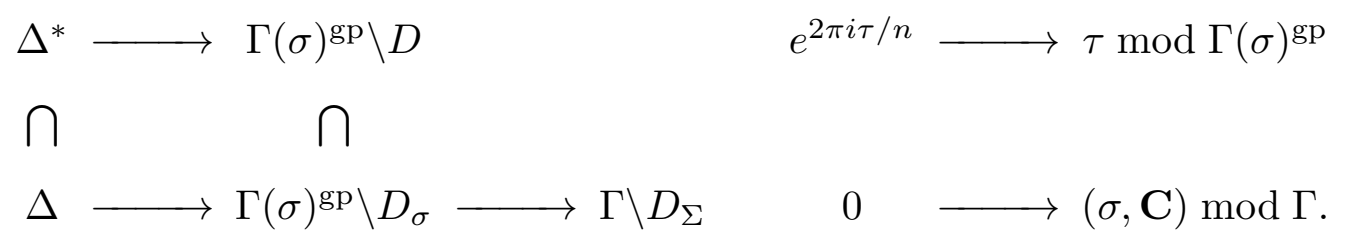

2.2. Tate type $([\mathrm{KNU} 12,7.1 .1])$

Let $H_{0}:=\mathbf{Z}^{2}=\mathbf{Z} e_{1}+\mathbf{Z} e_{2}$,

$$
W_{-3}=0 \subset W_{-2}=W_{-1}=\mathbf{R} e_{1} \subset W_{0}=W_{-1}+\mathbf{R} e_{2}=H_{0, \mathbf{R}},
$$

and $D$ the classifying space of mixed Hodge structures with weight filtration $W$. Then, $D=\mathbf{C}$ via $F=F(z) \leftrightarrow z$ where

$$
F^{1}:=0 \subset F^{0}:=\mathbf{C}\left(z e_{1}+e_{2}\right) \subset F^{-1}:=F^{0}+\mathbf{C} e_{1}=H_{0, \mathbf{C}}
$$

Let $\Gamma:=\left(\begin{array}{ll}1 & \mathbf{Z} \\ 0 & 1\end{array}\right), \Sigma=\{\sigma,-\sigma,\{0\}\}$ where $\pm \sigma:=\left(\begin{array}{cc}1 & \pm \mathbf{R}_{\geq 0} \\ 0 & 1\end{array}\right)$. Then, $\Gamma \backslash D=\mathbf{C}^{\times} \subset$ $\Gamma \backslash D_{\Sigma}=\mathbf{P}^{1}(\mathbf{C})$ via $(\sigma, D) \leftrightarrow 0$ and $(-\sigma, D) \leftrightarrow \infty$.

$\mathbf{Z}$-structure and model.

Let $S \in \mathcal{B}(\log )$. Then, $\mathcal{E} x t_{\mathrm{LMH} / S}^{1}(\mathbf{Z}, \mathbf{Z}(1))=M_{S}^{\mathrm{gp}}$ as functors on $\mathcal{B}(\log )$. In fact, for $a \in \Gamma\left(T, M_{T}^{\mathrm{gp}}\right)(T \in \mathcal{B}(\log ) / S)$, the corresponding object $H$ on $T$ of the left side functor is defined as follows. Let $\tau: T^{\log } \rightarrow T$ be as in 1.2 and let $0 \rightarrow \mathbf{Z}(1) \rightarrow \mathcal{L}_{T} \rightarrow$ $\tau^{-1} M_{T}^{\mathrm{gp}} \rightarrow 0$ be the exact sequence of the sheaf of logarithm from 1.2. Pulling back this sequence by $\mathbf{Z} \rightarrow \tau^{-1} M_{T}^{\mathrm{gp}}, 1 \mapsto a^{-1}$, we have the extension $0 \rightarrow \mathbf{Z}(1) \rightarrow H_{\mathbf{Z}} \rightarrow \mathbf{Z} \rightarrow 0$ of $\mathbf{Z}$-structures. The Hodge filtration is defined by $F^{0}:=\operatorname{Ker}\left(\mathcal{O}_{T}^{\log } \otimes H_{\mathbf{Z}} \stackrel{c}{\rightarrow} \mathcal{O}_{T}^{\log }\right)$, where $c$ is the morphism induced by $H_{\mathbf{Z}} \rightarrow \mathcal{L}_{T} \subset \mathcal{O}_{T}^{\text {log }}$.

This functor $\mathcal{E}_{x} t_{\mathrm{LMH} / S}^{1}(\mathbf{Z}, \mathbf{Z}(1))=M_{S}^{\mathrm{gp}}$ is not representable. But the subfunctor $T \mapsto \Gamma\left(T, M_{T} \cup M_{T}^{-1}\right)$ of $T \mapsto \Gamma\left(T, M_{T}^{\mathrm{gp}}\right)$ is represented by $\Gamma \backslash D_{\Sigma}=\mathbf{P}^{1}(\mathbf{C})$ ( $\Sigma$ as above).

\subsection{Mirror quintic}

Let $w=3, h^{p, q}=1(p+q=3, p, q \geq 0)$, and $h^{p, q}=0$ otherwise. Let $H_{0}=$ $\bigoplus_{j=1}^{4} \mathbf{Z} e_{j}=\mathbf{Z}^{4}$, and $\left\langle e_{3}, e_{1}\right\rangle_{0}=\left\langle e_{4}, e_{2}\right\rangle_{0}=1$. Let $D$ be the corresponding classifying space of polarized Hodge structures, and $\check{D}$ the "compact dual".

Let $S=$ (square free positive integers), and let $m \in S$. Define $N_{\alpha}, N_{\beta}, N_{m} \in$ $\operatorname{End}\left(H_{0},\langle,\rangle_{0}\right)$ as follows.

$$
\begin{aligned}
& N_{\alpha}\left(e_{3}\right)=e_{1}, \quad N_{\alpha}\left(e_{j}\right)=0 \quad(j \neq 3) ; \\
& N_{\beta}\left(e_{4}\right)=e_{3}, \quad N_{\beta}\left(e_{3}\right)=-e_{1}, \quad N_{\beta}\left(e_{1}\right)=-e_{2}, \quad N_{\beta}\left(e_{2}\right)=0 ; \\
& N_{m}\left(e_{1}\right)=e_{3}, \quad N_{m}\left(e_{4}\right)=-m e_{2}, \quad N_{m}\left(e_{2}\right)=N_{m}\left(e_{3}\right)=0 .
\end{aligned}
$$

Let $\sigma_{\alpha}=\mathbf{R}_{\geq 0} N_{\alpha}, \sigma_{\beta}=\mathbf{R}_{\geq 0} N_{\beta}$, and $\sigma_{m}=\mathbf{R}_{\geq 0} N_{m}(m \in S)$. 
Proposition ([KU09, 12.3]). Let $\Sigma=$ (rational nilpotent cones in $\mathfrak{g}_{\mathbf{R}}$ of rank $\left.\leq 1\right)$. Then $\Sigma=\left\{\operatorname{Ad}(g) \sigma \mid \sigma=\{0\}, \sigma_{\alpha}, \sigma_{\beta}, \sigma_{m}(m \in S), g \in G_{\mathbf{Q}}\right\}$. This is a complete fan, i.e., if there exists $Z \subset \check{D}$ such that $(\sigma, Z)$ is a nilpotent orbit, then $\sigma \in \Sigma$.

$\Gamma \backslash D_{\Sigma}$ is the complete fine moduli of log Hodge structures of specified type $\Phi=$ $\left(H_{0}, w,\langle,\rangle_{0},\left(h^{p, q}\right)_{p, q}, \Sigma, \Gamma\right)$ which receives the log period map for $H^{3}$ of mirror quintic 3 -folds. Indeed, the period map of mirror quintic 3-folds is uniquely extended over the compactification of the moduli and a generic global Torelli theorem for the period map of mirror quintic 3 -folds is proved by using the boundary points in [U08].

\subsection{Néron model}

We write here a relative version of the moduli space of LMH after [KNU12, 5-6].

Let $S$ be an object of $\mathcal{B}(\log )$, and assume that, for each $w \in \mathbf{Z}$, we are given a polarized log Hodge structure $H_{(w)}$ of weight $w$ on $S$. Assume $H_{(w)}=0$ for almost all $w$. Let $S^{\circ}$ be $S$ regarded as an object of $\mathcal{B}$ by forgetting the log structure, and $S^{\prime} \in \mathcal{B} / S^{\circ}$ is endowed with the pullback of the log structure of $S$.

Theorem $([\mathrm{KNU} 12,6.1 .1])$. In the above situation, there is a relative log manifold $J_{1}$ (resp. $J_{0}$ ) over $S$ which is strict over $S$, i.e., the log structure is the pullback of that of $S$, and which represents the following functor on $\mathcal{B} / S^{\circ}$ :

$\mathcal{B} / S^{\circ} \ni S^{\prime} \mapsto$ (the set of all isomorphism classes of LMH $H$ on $S^{\prime}$ with $H\left(\mathrm{gr}_{w}^{W}\right)=$ $H_{(w)}(w \in \mathbf{Z})$ satisfying the following condition: Locally on $S^{\prime}$, there is a splitting $H_{\mathbf{Q}}\left(\mathrm{gr}^{W}\right) \simeq H_{\mathbf{Q}}$ (resp. $\left.H_{\mathbf{Z}}\left(\mathrm{gr}^{W}\right) \simeq H_{\mathbf{Z}}\right)$ of the weight filtration $W$ on the local system $H_{\mathbf{Q}}\left(\operatorname{resp} . H_{\mathbf{Z}}\right)$ on $\left.\left(S^{\prime}\right)^{\log }\right)$.

We specialize the case. Let $w$ be a negative integer. Assume $H_{(k)}=0$ for $k \neq w, 0$, and that $H_{(0)}=\mathbf{Z}$. Let $H^{\prime}=H_{(w)}$. Consider the exact sequence on $S^{\text {log }}$

$$
0 \rightarrow H_{\mathbf{Z}}^{\prime} \rightarrow H_{\mathcal{O}^{\log }}^{\prime} / F^{0} \rightarrow H_{\mathbf{Z}}^{\prime} \backslash H_{\mathcal{O}^{\log }}^{\prime} / F^{0} \rightarrow 0 \text {. }
$$

Descending this by $\tau: S^{\log } \rightarrow S$, we have an exact sequence of sheaves of abelian groups on $\mathcal{B}(\log ) / S$

$$
0 \rightarrow \tau_{*} H_{\mathbf{Z}}^{\prime} \backslash\left(H_{\mathcal{O}}^{\prime} / F^{0} H_{\mathcal{O}}^{\prime}\right)^{s G} \rightarrow \mathcal{E} x t^{1}\left(\mathbf{Z}, H^{\prime}\right) \rightarrow R^{1} \tau_{*} H_{\mathbf{Z}}^{\prime}
$$

Here ()$^{s G}$ is the part restricted by the small Griffiths transversality (1.3). $\mathcal{E} x t^{1}\left(\mathbf{Z}, H^{\prime}\right)=$ $\mathcal{E} x t_{\mathrm{LMH} / S}^{1}\left(\mathbf{Z}, H^{\prime}\right)$ is the sheaf functor

$$
\mathcal{B}(\log ) / S \ni S^{\prime} \mapsto \operatorname{Ext}_{\mathrm{LMH} / S^{\prime}}^{1}\left(\mathbf{Z},\left.H^{\prime}\right|_{S^{\prime}}\right),
$$

where the image is the set of isomorphism classes of log mixed Hodge structure $\mathrm{H}$ on $S^{\prime}$ such that $\left.0 \rightarrow H^{\prime}\right|_{S^{\prime}} \rightarrow H \rightarrow \mathbf{Z} \rightarrow 0$ is exact in the category of log mixed Hodge structures, i.e., exact of lattice, of weight filtration, and of Hodge filtration. $\mathcal{E} x t^{1}\left(\mathbf{Z}, H^{\prime}\right)$ is identified, via $H \mapsto e_{F}-e_{\mathbf{Z}}$, with the subgroup of $\tau_{*}\left(H_{\mathbf{Z}}^{\prime} \backslash H_{\mathcal{O}^{\log }}^{\prime} / F^{0}\right)^{s G}$ restricted by the condition of the admissibility of local monodromy. 
Proposition ([KNU12, 6.1.4]). In the above situation:

(i) $O n \mathcal{B} / S^{\circ}$, the Néron model represents

$$
\operatorname{Ker}\left(\mathcal{E} x t^{1}\left(\mathbf{Z}, H^{\prime}\right) \rightarrow R^{1} \tau_{*} H_{\mathbf{Z}}^{\prime} \rightarrow R^{1} \tau_{*} H_{\mathbf{Q}}^{\prime}\right) .
$$

(ii) On $\mathcal{B} / S^{\circ}$, the connected Néron model represents

$$
\operatorname{Ker}\left(\mathcal{E} x t^{1}\left(\mathbf{Z}, H^{\prime}\right) \rightarrow R^{1} \tau_{*} H_{\mathbf{Z}}^{\prime}\right)=\tau_{*} H_{\mathbf{Z}}^{\prime} \backslash\left(H_{\mathcal{O}}^{\prime} / F^{0} H_{\mathcal{O}}^{\prime}\right)^{s G}
$$

We further specialize the case. Assume that $S$ is a complex analytic manifold with a smooth divisor which gives the $\log$ structure of $S$, and denote by $S^{*}$ the complement of the divisor in $S$. Assume $w=-1$.

Corollary ([KNU12, 6.1.6, 6.1.8]). In the above situation, the Néron model $J_{1}$ represents $\mathcal{E} x t^{1}\left(\mathbf{Z}, H^{\prime}\right)$ on $\mathcal{B} / S^{\circ}$, and we have

$$
\text { (a section of the Néron model over } S \text { ) }
$$

$=\left(\right.$ a normal function on $S^{*}$ which is admissible with respect to $S$ ).

\section{Quintic threefolds}

We recall some results for A-model of quintic threefold and B-model of mirror quintic mainly using the notation from [CoK99]. We introduce $\mathbf{Z}$-structure on $S^{\text {log }}$, and prove the equivalence of the formulation 0 (4) with the other formulations (1)-(3) in Introduction. We notice that $N$ in Section 3 is $-N$ of $N$ in Sections $1-2$.

\subsection{Picard-Fuchs ideal}

We review here [CoK99, 5.1.2]. Let $X \rightarrow S$ be a proper smooth family of Kähler manifolds of dimension $n$ over a smooth base $S$, and let $H$ be the variation of Hodge structure over $S$ arising from this family on the $n$-th relative cohomology sheaf. Let $\mathcal{D}$ be the sheaf of rings of holomorphic differential operators on $S$. The Gauss-Manin connection $\nabla$ on $H$ gives the $\mathcal{D}$-module structure on $H$. Let $\Omega$ be a fixed local section of the Hodge filter $F^{n}$ at a point $p \in S$. Let $z_{1}, \ldots, z_{r}$ be local coordinates of $S$ at $p$. Then, locally on $S, \mathcal{D}=\mathbf{C}\left\{z_{1}, \ldots, z_{r}\right\}\left[\partial / \partial z_{1}, \ldots, \partial / \partial z_{r}\right]$ and the Gauss-Manin connection $\nabla$ define an $\mathcal{O}_{S}$-homomorphism

$$
\phi: \mathcal{D} \rightarrow F^{0} \quad \text { by } \quad \phi\left(X_{1} \cdots X_{m}\right):=\nabla_{X_{1}} \cdots \nabla_{X_{m}} \Omega
$$

for vector fields $X_{i}$ on $S$. The ideal $I:=\operatorname{Ker}(\phi)$ is called the Picard-Fuchs ideal. Since

$$
\frac{\partial}{\partial z_{j}} \int_{\gamma} \Omega=\int_{\gamma} \nabla_{\partial / \partial z_{j}} \Omega
$$


for a locally constant homology $n$-cycle $\gamma$, the periods $y:=\int_{\gamma} \Omega$ of $\Omega$ are solutions of the equations $D y=0$ for all $D \in I$.

By analytic continuation, the above things make sense as algebraic differential equations over all $S$ if we restrict our attention to the periods of algebraic differentials.

\subsection{Quintic threefold and its mirror}

Let $V$ be a generic quintic threefold in $\mathbf{P}^{4}$. Let $V_{\psi}$ be a hypersurface in $\mathbf{P}^{4}$ defined by $f:=x_{1}^{5}+x_{2}^{5}+x_{3}^{5}+x_{4}^{5}+x_{5}^{5}+\psi x_{1} x_{2} x_{3} x_{4} x_{5}=0\left(\psi \in \mathbf{P}^{1}\right)$. Let $G:=\left\{\left(a_{j}\right) \in\right.$ $\left.\left(\mu_{5}\right)^{5} \mid a_{1} \ldots a_{5}=1\right\}$. Under the coordinate-wise action, $x_{j} \mapsto a_{j} x_{j}$, take the quotient $V_{\psi} / G$. Then a mirror to $V$ is given by a resolution $V_{\psi}^{\circ}$ of the quotient singularity of $V_{\psi} / G$ (see [M93], [CoK99]).

\subsection{Picard-Fuchs equation on the mirror $V^{\circ}$}

Let $\Omega$ be the holomorphic 3-form on the mirror $V_{\psi}^{\circ}$ induced from the one

$$
\operatorname{Res}_{V_{\psi}}\left(\frac{\psi}{f} \sum_{j=1}^{5}(-1)^{j-1} x_{j} d x_{1} \wedge \cdots \wedge\left(d x_{j}\right)^{\wedge} \wedge \cdots \wedge d x_{5}\right)
$$

on $V_{\psi}$. Let $z:=1 / \psi^{5}$ and $\delta:=z d / d z$. For $\Omega$, the Picard-Fuchs equation near $z=0$ is

$$
\begin{aligned}
& L:=\delta^{4}+5 z(5 \delta+1)(5 \delta+2)(5 \delta+3)(5 \delta+4), \\
& L y=\left(\delta^{4}+\frac{2 \cdot 5^{5} z}{1+5^{5} z} \delta^{3}+\frac{7 \cdot 5^{4} z}{1+5^{5} z} \delta^{2}+\frac{2 \cdot 5^{4} z}{1+5^{5} z} \delta+\frac{24 \cdot 5 z}{1+5^{5} z}\right) y=0
\end{aligned}
$$

(cf. [CoK99, 5.4]), and its indicial equation at $z=0$ is $\rho^{4}=0$, i.e., maximally unipotent. By the Frobenius method, we have a basis of solutions $y_{i}(z)(0 \leq i \leq 3)$ as follows. Let

$$
\tilde{y}(-z ; \rho):=\sum_{n=0}^{\infty} \frac{\prod_{m=1}^{5 n}(5 \rho+m)}{\prod_{m=1}^{n}(\rho+m)^{5}}(-z)^{n+\rho}
$$

be a solution of $L(\tilde{y}(-z ; \rho))=\rho^{4}(-z)^{\rho}$, and let

$$
\tilde{y}(-z ; \rho)=y_{0}(z)+y_{1}(z) \rho+y_{2}(z) \rho^{2}+y_{3}(z) \rho^{3}+\cdots, \quad y_{i}(z):=\left.\frac{1}{i !} \frac{\partial^{i} \tilde{y}(-z ; \rho)}{\partial \rho^{i}}\right|_{\rho=0}
$$

be the Taylor expansion at $\rho=0$. The first two functions of the basis are computed as

$$
\begin{aligned}
& y_{0}=\sum_{n=0}^{\infty} \frac{(5 n) !}{(n !)^{5}}(-z)^{n} \\
& y_{1}=y_{0} \log (-z)+5 \sum_{n=1}^{\infty} \frac{(5 n) !}{(n !)^{5}}\left(\sum_{j=n+1}^{5 n} \frac{1}{j}\right)(-z)^{n} .
\end{aligned}
$$


Let $t:=y_{1} / y_{0}$ and $u:=t / 2 \pi i$. The canonical coordinate is given by $q:=e^{t}=e^{2 \pi i u}$ which is a specific chart of the log structure and gives the mirror map.

The Gauss-Manin potential of the mirror $V_{\psi}^{\circ}$ is given by

$$
\Phi_{\mathrm{GM}}^{V^{\circ}}=\frac{5}{2}\left(\frac{y_{1}}{y_{0}} \frac{y_{2}}{y_{0}}-\frac{y_{3}}{y_{0}}\right)
$$

(cf. [CoK99, Theorem 8.6.5]).

[CDGP91] computed the above complete solutions of the Picard-Fuchs equation by using Gamma function, their analytic continuations, and local monodoromies around $z=0, \infty$, and $-1 / 5^{5}$. The Yukawa coupling at the maximally unipotent point $z=0$ is given by

$$
Y:=-\int_{V^{\circ}} \tilde{\Omega} \wedge \nabla_{\delta} \nabla_{\delta} \nabla_{\delta} \tilde{\Omega}=\frac{5}{\left(1+5^{5} z\right) y_{0}(z)^{2}}\left(\frac{q}{z} \frac{d z}{d q}\right)^{3},
$$

where $\tilde{\Omega}:=\Omega / y_{0}$ is the normalized holomorphic 3-form (cf. [CoK99, Example 5.6.4.1]).

\subsection{A-model of the quintic $V$}

Let $V$ be a generic quintic threefold. Then $\operatorname{Aut}(V)=\{1\}$. Let $T_{1}:=H$ be the hyperplane section of $V$ in $\mathbf{P}^{4}$ and let $K(V)=\mathbf{R}_{>0} T_{1}$ be the Kähler cone of $V$. Let $u$ be the coordinate of $\mathbf{C} T_{1}$. The complexified Kähler moduli $\mathcal{K} \mathcal{M}(V)$ of $V$ is isomorphic to the punctured disc:

$$
\mathcal{K} \mathcal{M}(V)=\left(H^{2}(V, \mathbf{R})+i K(V)\right) / \text { Image } H^{2}(V, \mathbf{Z}) \stackrel{\sim}{\rightarrow} \Delta^{*} \subset \Delta, \quad u T_{1} \mapsto q:=\exp (2 \pi i u) .
$$

The basis of $H_{2}(V, \mathbf{Z}) \simeq \mathbf{Z}$ is given by the homology class $C$ of the line on $V$. Let $T^{1}$ be the cohomology class of the line, i.e., the Poincaré dual of $C$. For $\beta=d C \in H_{2}(V, \mathbf{Z})$ $(d \in \mathbf{Z})$, define $q^{\beta}:=q^{\int_{\beta} T^{1}}=q^{d}$. The Gromov-Witten potential of $V$ is defined by

$$
\begin{aligned}
\Phi_{\mathrm{GW}}^{V} & :=\frac{1}{6} \int_{V}\left(t T_{1}\right)^{3}+\sum_{0 \neq \beta \in H_{2}(V, \mathbf{Z})}\left\langle I_{0,0, \beta}\right\rangle q^{\beta} \\
& =\frac{5 t^{3}}{6}+\sum_{d>0}\left\langle I_{0,0, d}\right\rangle q^{d}=\frac{5(2 \pi i u)^{3}}{6}+\sum_{d>0}\left\langle I_{0,0, d}\right\rangle q^{d}
\end{aligned}
$$

([CoK99, (8.51)]), where $t=2 \pi i u$. Here $N_{d}:=\left\langle I_{0,0, d}\right\rangle$ is the Gomov-Witten invariant defined as follows. Fix a positive integer $d$ and consider the diagram of moduli spaces of stable curves

$$
\bar{M}_{0,0}\left(\mathbf{P}^{4}, d\right) \stackrel{\pi_{1}}{\longleftarrow} \bar{M}_{0,1}\left(\mathbf{P}^{4}, d\right) \stackrel{e_{1}}{\longrightarrow} \mathbf{P}^{4}
$$

where $\pi_{1}$ forgets the marked point and $e_{1}$ is the evaluation map. Define

$$
\left\langle I_{0,0, d}\right\rangle:=\int_{\bar{M}_{0,0}\left(\mathbf{P}^{4}, d\right)} c_{5 d+1}\left(\pi_{1 *} e_{1}^{*} \mathcal{O}_{\mathbf{P}^{4}}(5)\right) .
$$


We have $\left\langle I_{0,0, d}\right\rangle=\sum_{k \mid d} n_{d / k} k^{-3}$, where $n_{d / k}$ is the instanton number (see, e.g., [CoK99, 11.1.1]). $N_{d}=0$ if $d \leq 0$.

\subsection{Z-structure}

We first consider B-model $\mathcal{H}^{V^{\circ}}$ of quintic-mirror from (3) in Introduction.

Let $f: X \rightarrow S^{*}$ be a smooth projective family of quintic-mirrors over a punctured neighborhood of the maximally unipotent point. Let $\mathcal{H}_{\mathbf{Z}}^{V^{\circ}}=R^{3} f_{*} \mathbf{Z}$, and extend this to the local system of $\mathbf{Z}$-modules over $S^{\log }$ (cf. [KU09, 2.3.5], [O03], [U01]), still denoted $\mathcal{H}_{\mathbf{Z}}^{V^{\circ}}$. Let $N$ be the monodromy logarithm at the maximally unipotent point, and $W=W(N)$ be the monodromy weight filtration. Define $W_{k, \mathbf{Z}}:=W_{k} \cap \mathcal{H}_{\mathbf{Z}}^{V^{\circ}}$ for all $k$.

Take a point $b \in S^{\log }$ and fix it. We claim that there is a symplectic $\mathbf{Z}$-basis $g_{0}, g_{1}, g_{3}, g_{2}$ of $\mathcal{H}_{\mathbf{Z}}^{V^{\circ}}(b)$ for the cup product $\langle\mid\rangle$ which gives a splitting of the filtration $W$, i.e., $g_{0}, \ldots, g_{k}$ generate $W_{2 k}$ for all $k$, and $\left\langle g_{0} \mid g_{3}\right\rangle=1,\left\langle g_{1} \mid g_{2}\right\rangle=1,\left\langle g_{0} \mid g_{j}\right\rangle=0$ for $j \neq 3,\left\langle g_{1} \mid g_{j}\right\rangle=0$ for $j \neq 2$. This claim follows from [M93]. In fact, let $g_{0}, g_{1}$ be a good integral basis of $W_{2}(b)$ in the sense of $[\mathrm{M} 93,2]$, i.e., $g_{0}$ is a generator of $W_{0, \mathbf{Z}}(b)$, and $g_{1}$ is an indivisible element of $\mathcal{H}_{\mathbf{Z}}^{V^{\circ}}(b)$ which can be written as $g_{1}=(1 / \lambda) N^{2} g$ for some $\lambda>0$ and some $g \in \mathcal{H}_{\mathrm{Z}}^{V^{\circ}}(b)$ such that $\left\langle g_{0} \mid g\right\rangle=1$. Put $g_{3}:=g$. Since the cup product is skew-symmetric unimodular, we can take $g_{2}^{\prime} \in \mathcal{H}_{\mathbf{Z}}^{V^{\circ}}(b)$ such that $\left\langle g_{1} \mid g_{2}^{\prime}\right\rangle=1$. Let $g_{2}:=g_{2}^{\prime}-\left\langle g_{0} \mid g_{2}^{\prime}\right\rangle g_{3}+\left\langle g_{3} \mid g_{2}^{\prime}\right\rangle g_{0}$. Then, $g_{0}, g_{1}, g_{2}, g_{3}$ is a desired basis.

Log Hodge theoretic description of $\mathcal{H}_{\mathbf{Z}}^{V^{\circ}}$ is as follows. Let $\left(S^{\log }, \mathcal{O}_{S}^{\log }\right)$ be the ringed space from 1.2. Then, for a local section $s \in \mathcal{O}_{S}^{\log } \otimes_{\mathcal{O}} \mathcal{H}_{\mathcal{O}}^{V^{\circ}}$, the following statements are equivalent:

(1) $s$ belongs to $\mathcal{H}_{\mathrm{Z}}^{V^{\circ}}$

(2) $\nabla s=0\left(\nabla=\nabla^{\mathrm{GM}}\right)$ and $s(b) \in \mathcal{H}_{\mathbf{Z}}^{V^{\circ}}(b)$ for some $b \in S^{\log }$.

(3) $\nabla s=0$ and $s\left(\operatorname{gr}_{k}^{W}\right) \in \operatorname{gr}_{k, \mathbf{Z}}^{W}$ for $k:=\min \left\{l \mid s \in \mathcal{O}_{S}^{\log } \otimes W_{l}\right\}$.

Now we consider A-model $\mathcal{H}^{V}$ of quintic threefold from (3) in Introduction.

Let $\nabla=\nabla^{\text {middle }}$ be the A-model connection from 3.6 (3A) below. For $s \in \mathcal{O}_{S}^{\log } \otimes_{\mathcal{O}}$ $\mathcal{H}_{\mathcal{O}}^{V}$, define $s \in \mathcal{H}_{\mathbf{Z}}^{V}$ if $\nabla s=0$ and $s\left(\operatorname{gr}_{2 p}^{W}\right) \in H^{3-p, 3-p}(V, \mathbf{Z})$ for $p:=\min \{q \mid s \in$ $\left.\mathcal{O}_{S}^{\log } \otimes W_{2 q}\right\}$. Here $W_{2 q}:=\bigoplus_{l \leq q} H^{3-l, 3-l}(V, \mathbf{R})$.

For log Hodge theoretic description, we recall the following. Let $0 \in S=\Delta$ and $b \in \tau^{-1}(0) \subset S^{\log }$. From 1.2, the stalk over $b$ is $\mathcal{O}_{S, b}^{\log }=\mathcal{O}_{S, 0}[t]=\mathbf{C}\{q\}[t]$ which is a polynomial ring in $t$ over the convergent power series in $q$, where $q=e^{2 \pi i u}=e^{t}$ from 3.4. Let $u=x+i y$ with $x, y$ real.

For $s \in \mathcal{O}_{S}^{\log } \otimes_{\mathcal{O}} \mathcal{H}_{\mathcal{O}}^{V}$, the following statements are equivalent:

(4) $s$ belongs to $\mathcal{H}_{\mathrm{Z}}^{V}$.

(5) $\nabla s=0$ and $s(b) \in \mathcal{H}_{\mathbf{Z}}^{V}(b)$ for some $b \in S^{\log }$.

(6) $\nabla s=0$ and, for fixed $x=0$, the limit of $\exp (i y(-N)) s$ over $S^{\log }$ belongs to $\bigoplus_{p} H^{p, p}(V, \mathbf{Z})$.

(7) $\nabla s=0$ and the specialization $x \mapsto 0$ of the limit of $\exp (i y(-N)) s$ over $S^{\log }$ with $x$ fixed and $y \rightarrow \infty$ belongs to $\bigoplus_{p} H^{p, p}(V, \mathbf{Z})$. 
For computation of specific examples, see 3.7 below.

\subsection{Correspondence table}

The following is the correspondence table expanded the one from [CoK99, 8.5-8.6].

(1A) Polarization of $A$-model of $V$.

$$
S(\alpha, \beta):=(-1)^{p} \int_{V} \alpha \cup \beta \quad\left(\alpha \in H^{p, p}(V), \beta \in H^{3-p, 3-p}(V)\right) .
$$

(1B) Polarization of $B$-model of $V^{\circ}$.

$$
Q(\alpha, \beta):=(-1)^{3(3-1) / 2} \int_{V^{\circ}} \alpha \cup \beta=-\int_{V^{\circ}} \alpha \cup \beta \quad\left(\alpha, \beta \in H^{3}\left(V^{\circ}\right)\right) .
$$

(2A) Specified sections inducing Z-basis of graded quotients of A-model of $V$.

We take

$$
T_{0}:=1 \in H^{0}(V, \mathbf{Z}), T_{1}:=H \in H^{2}(V, \mathbf{Z}), T^{1}:=C \in H^{4}(V, \mathbf{Z}), T^{0}:=[p t] \in H^{6}(V, \mathbf{Z}),
$$

Then $S\left(T_{0}, T^{0}\right)=1$ and $S\left(T_{1}, T^{1}\right)=-1$. Hence $T_{0}, T_{1},-T^{0}, T^{1}$ form a symplectic base for $S$.

(2B) Specified sections inducing $\mathbf{Z}$-basis of graded quotients of B-model of $V^{\circ}$.

After [D97], we use the decomposition of the associated mixed Hodge structure of Hodge-Tate type $(\mathcal{W}, \mathcal{F})$ :

$$
\mathcal{H}_{\mathcal{O}}=\bigoplus_{p} I^{p, p}, \quad \text { where } \quad I^{p, p}:=\mathcal{W}_{2 p} \cap \mathcal{F}^{p} \stackrel{\sim}{\rightarrow} \operatorname{gr}_{2 p}^{\mathcal{W}}
$$

Since $N\left(\operatorname{gr}_{2 p}^{W}\right)=0, \operatorname{gr}_{2 p}^{W}$ is a constant sheaf and hence

$$
\operatorname{gr}_{2 p}^{\mathcal{W}} \supset \operatorname{gr}_{2 p}^{W} \supset\left(\operatorname{gr}_{2 p}^{W}\right) \mathbf{z}:=W_{2 p, \mathbf{Z}} / W_{2 p-1, \mathbf{Z}}
$$

Now we can take

$$
e_{0}:=\tilde{\Omega} \in I^{3,3}, e_{1} \in I^{2,2}, e^{1} \in I^{1,1}, e^{0}=g_{0} \in I^{0,0}
$$

inducing generators of $\left(\mathrm{gr}_{2 p}^{W}\right) \mathbf{z}$ and satisfying $Q\left(e_{0}, e^{0}\right)=1$ and $Q\left(e_{1}, e^{1}\right)=-1$. Hence $e_{0}, e_{1},-e^{0}, e^{1}$ form a symplectic base for $Q$.

(3A) A-model connection $\nabla=\nabla^{\text {middle }}$ of $V$.

For $\delta:=d / d u$, A-model connection is defined by

$$
\begin{aligned}
\nabla_{\delta} T^{0} & :=0, \quad \nabla_{\delta} T^{1}:=T^{0} \\
\nabla_{\delta} T_{1} & :=\frac{1}{(2 \pi i)^{3}} \frac{d^{3} \Phi}{d u^{3}} T^{1}=\left(5+\frac{1}{(2 \pi i)^{3}} \frac{d^{3} \Phi_{\mathrm{hol}}}{d u^{3}}\right) T^{1} \\
\nabla_{\delta} T_{0} & :=T_{1} .
\end{aligned}
$$


$\nabla$ is flat, i.e., $\nabla^{2}=0$ (cf. [CoK99, Prop. 8.5.2]).

(3B) B-model connection $\nabla=\nabla^{\mathrm{GM}}$ of $V^{\circ}$.

For $\delta:=d / d u$, the Gauss-Manin connection is computed in [CDGP91] as

$$
\begin{aligned}
& \nabla_{\delta} e^{0}=0, \quad \nabla_{\delta} e^{1}=e^{0} \\
& \nabla_{\delta} e_{1}=\frac{1}{(2 \pi i)^{3}} \frac{d^{3} \Phi}{d u^{3}} e^{1}=Y e^{1}=\frac{5}{\left(1+5^{5}\right) y_{0}(z)^{2}}\left(\frac{q}{z} \frac{d z}{d q}\right)^{3} e^{1} \\
& \nabla_{\delta} e_{0}=e_{1}
\end{aligned}
$$

(4A) $\nabla$-flat $\mathbf{Z}$-basis for $\mathcal{H}_{\mathbf{Z}}^{V}$.

It is computed as

$$
\begin{aligned}
s^{0}: & =T^{0} \\
s^{1}: & =T^{1}-u T^{0}=\exp (-u H) T^{1}, \\
s_{1}:= & T_{1}-\frac{1}{(2 \pi i)^{3}} \frac{d^{2} \Phi}{d u^{2}} T^{1}+\frac{1}{(2 \pi i)^{3}} \frac{d \Phi}{d u} T^{0} \\
= & \exp (-u H) T_{1}-\left(\sum_{d>0} \frac{N_{d} d^{2}}{2 \pi i} q^{d}\right) T^{1}+\left(\sum_{d>0} \frac{N_{d} d}{(2 \pi i)^{2}} q^{d}\right) T^{0}, \\
s_{0}:= & T_{0}-u T_{1}+\frac{1}{(2 \pi i)^{3}}\left(u \frac{d^{2} \Phi}{d u^{2}}-\frac{d \Phi}{d u}\right) T^{1}-\frac{1}{(2 \pi i)^{3}}\left(u \frac{d \Phi}{d u}-2 \Phi\right) T^{0} \\
= & \exp (-u H) T_{0}+\left(\sum_{d>0} \frac{N_{d} d^{2}}{2 \pi i} u q^{d}-\sum_{d>0} \frac{N_{d} d}{(2 \pi i)^{2}} q^{d}\right) T^{1} \\
& -\left(\sum_{d>0} \frac{N_{d} d}{(2 \pi i)^{2}} u q^{d}-\sum_{d>0} \frac{2 N_{d}}{(2 \pi i)^{3}} q^{d}\right) T^{0}
\end{aligned}
$$

(4B) $\nabla$-flat $\mathbf{Z}$-basis for $\mathcal{H}_{\mathbf{Z}}^{V^{\circ}}$.

It is computed as

$$
\begin{aligned}
& s^{0}:=e^{0}, \\
& s^{1}:=e^{1}-u e^{0}, \\
& s_{1}:=e_{1}-\frac{1}{(2 \pi i)^{3}} \frac{d^{2} \Phi}{d u^{2}} e^{1}+\frac{1}{(2 \pi i)^{3}} \frac{d \Phi}{d u} e^{0}, \\
& s_{0}:=e_{0}-u e_{1}+\frac{1}{(2 \pi i)^{3}}\left(u \frac{d^{2} \Phi}{d u^{2}}-\frac{d \Phi}{d u}\right) e^{1}-\frac{1}{(2 \pi i)^{3}}\left(u \frac{d \Phi}{d u}-2 \Phi\right) e^{0} .
\end{aligned}
$$

This is an explicit description of the $\mathbf{Z}$-basis of $\mathcal{H}_{\mathbf{Z}}^{V^{\circ}}$ (3.5) by using the basis from (2B).

(5A) Monodromy logarithm for A-model of $V$ around the large radius limit point. 
It is computed as

$$
N s^{0}=0, \quad N s^{1}=-s^{0}, \quad N s_{1}=-5 s^{1}, \quad N s_{0}=-s_{1} .
$$

Note that the matrix of monodromy logarithm $N$ via the basis $s^{0}, s^{1}, s_{1}, s_{0}$ coincides with the matrix of cup product with $-H$ via the basis $T^{0}, T^{1}, T_{1}, T_{0}(2 \mathrm{~A})$.

(5B) Monodromy logarithm for B-model of $V^{\circ}$ around the maximally unipotent monodromy point.

It is computed as

$$
N s^{0}=0, \quad N s^{1}=-s^{0}, \quad N s_{1}=-5 s^{1}, \quad N s_{0}=-s_{1} .
$$

(6A) Expression of $T^{i}, T_{i}$ by $s^{i}, s_{i}$.

It is computed that $T^{i}, T_{i}$ are written by the $\nabla$-flat $\mathbf{Z}$-basis $s^{i}, s_{i}$ of $\mathcal{H}_{\mathbf{Z}}^{V}$ as follows.

$$
\begin{aligned}
T^{0}= & s^{0} \\
T^{1}= & s^{1}+u s^{0} \\
T_{1}= & s_{1}+\frac{1}{(2 \pi i)^{3}} \frac{d^{2} \Phi}{d u^{2}} s^{1}+\frac{1}{(2 \pi i)^{3}}\left(u \frac{d^{2} \Phi}{d u^{2}}-\frac{d \Phi}{d u}\right) s^{0} \\
= & \left(s_{1}+5 u s^{1}+\frac{5}{2} u^{2} s^{0}\right)+\left(\sum_{d>0} \frac{N_{d} d^{2}}{2 \pi i} q^{d}\right) s^{1}+\left(\sum_{d>0} \frac{N_{d} d^{2}}{2 \pi i} u q^{d}-\sum_{d>0} \frac{N_{d} d}{(2 \pi i)^{2}} q^{d}\right) s^{0} \\
T_{0}= & s_{0}+u s_{1}+\frac{1}{(2 \pi i)^{3}} \frac{d \Phi}{d u} s^{1}+\frac{1}{(2 \pi i)^{3}}\left(u \frac{d \Phi}{d u}-2 \Phi\right) s^{0} \\
= & \left(s_{0}+u s_{1}+\frac{5}{2} u^{2} s^{1}+\frac{5}{6} u^{3} s^{0}\right)+\left(\sum_{d>0} \frac{N_{d} d}{(2 \pi i)^{2}} q^{d}\right) s^{1} \\
& +\left(\sum_{d>0} \frac{N_{d} d}{(2 \pi i)^{2}} u q^{d}-2 \sum_{d>0} \frac{N_{d}}{(2 \pi i)^{3}} q^{d}\right) s^{0} .
\end{aligned}
$$

Note that the section $1=T_{0}$ varies with respect to the the lattice $\mathcal{H}_{\mathbf{Z}}^{V}$ as above while the section $[\mathrm{pt}]=T^{0}=s^{0}$ does not.

(6B) Expression of $e^{i}, e_{i}$ by $s^{i}, s_{i}$. 
It is computed that $e^{i}, e_{i}$ are written by the $\nabla$-flat $\mathbf{Z}$-basis $s^{i}, s_{i}$ of $\mathcal{H}_{\mathbf{Z}}^{V^{\circ}}$ as follows.

$$
\begin{aligned}
e^{0}= & s^{0} \\
e^{1}= & s^{1}+u s^{0} \\
e_{1}= & s_{1}+\frac{1}{(2 \pi i)^{3}} \frac{d^{2} \Phi}{d u^{2}} s^{1}+\frac{1}{(2 \pi i)^{3}}\left(u \frac{d^{2} \Phi}{d u^{2}}-\frac{d \Phi}{d u}\right) s^{0} \\
= & \left(s_{1}+5 u s^{1}+\frac{5}{2} u^{2} s^{0}\right)+\left(\sum_{d>0} \frac{N_{d} d^{2}}{2 \pi i} q^{d}\right) s^{1}+\left(\sum_{d>0} \frac{N_{d} d^{2}}{2 \pi i} u q^{d}-\sum_{d>0} \frac{N_{d} d}{(2 \pi i)^{2}} q^{d}\right) s^{0} \\
e_{0}= & s_{0}+u s_{1}+\frac{1}{(2 \pi i)^{3}} \frac{d \Phi}{d u} s^{1}+\frac{1}{(2 \pi i)^{3}}\left(u \frac{d \Phi}{d u}-2 \Phi\right) s^{0} \\
= & \left(s_{0}+u s_{1}+\frac{5}{2} u^{2} s^{1}+\frac{5}{6} u^{3} s^{0}\right)+\left(\sum_{d>0} \frac{N_{d} d}{(2 \pi i)^{2}} q^{d}\right) s^{1} \\
& +\left(\sum_{d>0} \frac{N_{d} d}{(2 \pi i)^{2}} u q^{d}-2 \sum_{d>0} \frac{N_{d}}{(2 \pi i)^{3}} q^{d}\right) s^{0} \\
= & s_{0}+\frac{1}{2 \pi i} \frac{y_{1}}{y_{0}} s_{1}+\frac{5}{(2 \pi i)^{2}} \frac{y_{2}}{y_{0}} s^{1}+\frac{5}{(2 \pi i)^{3}} \frac{y_{3}}{y_{0}} s^{0} .
\end{aligned}
$$

Note that the normalized holomorphic 3 -form $\tilde{\Omega}:=\Omega / y_{0}=e_{0}$ varies with respect to the the lattice $\mathcal{H}_{\mathbf{Z}}^{V^{\circ}}$ as above, while the section $g_{0}=e^{0}=s^{0}$ does not.

(7A) A-model monodromy weight filtration of $\mathcal{H}^{V}$.

It is computed as follows, which does not vary.

$$
\begin{aligned}
& W_{0}=\mathbf{R}[\mathrm{pt}]=\mathbf{R} T^{0}=\mathbf{R} s^{0}=W_{1} \subset W_{2}=W_{1}+\mathbf{R} C=W_{1}+\mathbf{R} T^{1}=W_{3} \\
& \subset W_{4}=W_{3}+\mathbf{R} H=W_{3}+\mathbf{R} T_{1}=W_{5} \subset W_{6}=W_{5}+\mathbf{R} \cdot 1=W_{5}+\mathbf{R} T_{0} .
\end{aligned}
$$

(8A) A-model Hodge filtration of $\mathcal{H}^{V}$.

Let $\mathcal{F}^{p}:=F^{p} \times \mathcal{K} \mathcal{M}(V) \subset \mathcal{H}_{\mathcal{O}}^{V}$. This is computed as follows.

$$
\begin{aligned}
& \mathcal{F}^{3}=H^{0,0}(V)=\mathbf{C} \cdot 1=\mathbf{C} T_{0} \subset \mathcal{F}^{2}=\mathcal{F}^{3}+H^{1,1}(V)=\mathcal{F}^{3}+\mathbf{C H}=\mathcal{F}^{3}+\mathbf{C} T_{1} \\
& \subset \mathcal{F}^{1}=\mathcal{F}^{2}+H^{2,2}(V)=\mathcal{F}^{2}+\mathbf{C} C=\mathcal{F}^{2}+\mathbf{C} T^{1} \\
& \subset \mathcal{F}^{0}=\mathcal{F}^{1}+H^{3,3}(V)=\mathcal{F}^{1}+\mathbf{C}[\mathrm{pt}]=\mathcal{F}^{1}+\mathbf{C} T^{0} .
\end{aligned}
$$

Note that the mixed Hodge structure for A-model of $V$ from (7A) and (8A) is opposite to the original one from $(2 \mathrm{~A})$.

\subsection{Examples of computation}

Let $u=x+i y$ be as in 3.5 . 
First we compute for $s_{0}$ from 3.6 (4A) the specialization $x \mapsto 0$ of the limit of $\exp (i y(-N)) s_{0}$ over $S^{\log }$ with $x$ fixed and $y \rightarrow \infty$.

$$
\begin{aligned}
& \exp (i y(-N)) s_{0}=\left(T_{0}-x T_{1}+\frac{5}{2} x^{2} T^{1}-\frac{5}{6} x^{3} T^{0}\right) \\
& \quad+\left(\sum_{d>0} \frac{N_{d} d^{2}}{2 \pi i} x q^{d}-\sum_{d>0} \frac{N_{d} d}{(2 \pi i)^{2}} q^{d}\right) T^{1}+\left(2 \sum_{d>0} \frac{N_{d}}{(2 \pi i)^{3}} q^{d}-\sum_{d>0} \frac{N_{d} d}{(2 \pi i)^{2}} x q^{d}\right) T^{0} \\
& \stackrel{(x \text { fixed, } y \rightarrow \infty)}{\longrightarrow} T_{0}-x T_{1}+\frac{5}{2} x^{2} T^{1}-\frac{5}{6} x^{3} T^{0} \stackrel{(x \mapsto 0)}{\longrightarrow} T_{0} .
\end{aligned}
$$

We notice that $-N$ here in Section 3 is $N$ in Sections $1-2$.

If we use $\Phi_{\mathrm{GM}}^{V^{\circ}}=\Phi_{\mathrm{GW}}^{V}$, we have an analogous computation for $s_{0}$ from 3.6 (4B).

$$
\begin{aligned}
& \exp (i y(-N)) s_{0}=\left(e_{0}-x e_{1}+\frac{5}{2} x^{2} e^{1}-\frac{5}{6} x^{3} e^{0}\right) \\
& \quad+\left(\sum_{d>0} \frac{N_{d} d^{2}}{2 \pi i} x q^{d}-\sum_{d>0} \frac{N_{d} d}{(2 \pi i)^{2}} q^{d}\right) e^{1}+\left(2 \sum_{d>0} \frac{N_{d}}{(2 \pi i)^{3}} q^{d}-\sum_{d>0} \frac{N_{d} d}{(2 \pi i)^{2}} x q^{d}\right) e^{0} \\
& \stackrel{(x \text { fixed, } y \rightarrow \infty)}{\longrightarrow} e_{0}-x e_{1}+\frac{5}{2} x^{2} e^{1}-\frac{5}{6} x^{3} e^{0} \stackrel{(x \mapsto 0)}{\longrightarrow} e_{0} .
\end{aligned}
$$

We make a comment on the "value at $q=0$ of the canonical extension", e.g. $\exp (-u N) s_{0}$ for $s_{0}$ from $3.6(4 \mathrm{~A})$. It would be computed as

$$
\begin{aligned}
& \exp (-u N) s_{0}=s_{0}-(u N) s_{0}+\frac{1}{2}(u N)^{2} s_{0}-\frac{1}{6}(u N)^{3} s_{0} \\
& =s_{0}-u N s_{0}+\frac{1}{2} u^{2} N^{2} s_{0}-\frac{1}{6} u^{3} N^{3} s_{0}=s_{0}+u s_{1}+\frac{5}{2} u^{2} s^{1}+\frac{5}{6} u^{3} s^{0} \\
& =T_{0}+\left(\frac{5}{2} u^{2}-\frac{1}{(2 \pi i)^{3}} \frac{d \Phi}{d u}\right) T^{1}+\left(-\frac{5}{3} u^{3}+\frac{2}{(2 \pi i)^{3}} \Phi\right) T^{0} \\
& =T_{0}-\sum_{d>0} \frac{N_{d} d}{(2 \pi i)^{2}} q^{d} T^{1}+\sum_{d>0} \frac{2 N_{d}}{(2 \pi i)^{3}} q^{d} T^{0} \stackrel{(q \rightarrow 0)}{\longrightarrow} T_{0} .
\end{aligned}
$$

In this computation, it is allowed only to take a limit over the space $S^{\log }$ with fixed $x$ and $y \rightarrow \infty$. Then the $(u N)^{j}=u^{j} N^{j}$ hold in the second equality. If $u$ goes around the origin, it does not hold. This point is not clear in [CoK99] Proposition 5.6.1 and Proposition 8.5.4, their proofs, and the arguments after them.

\subsection{On equivalent statements (1)-(3) in Introduction}

The formulation 0 (1), i.e., coincidence of the potentials, is proved in [LLuY97], cf. [CoK99, 11.1, especially Theorem 11.1.1].

The formulation 0 (2), i.e., coincidence of the complete set of solutions, is announced and proved in [Gi96], [Gi97p], cf. [CoK99, 11.2, especially Example 11.2.1.3].

The formulation 0 (3), i.e., coincidence of polarized variation of Hodge structure with specified sections, is proved in [M97], cf. [CoK99, 8.6.1-8.6.2, especially Theorem 8.6.2]. 
Assuming $\Phi_{\mathrm{GW}}^{V}=\Phi_{\mathrm{GM}}^{V^{\circ}}$, we made the table in 3.6. The equations $3.6(6 \mathrm{~A})$ and $(6 \mathrm{~B})$ explain $0(2)$, and the equation $3.6(6 \mathrm{~B})$ explains $\Phi_{\mathrm{GM}}^{V^{\circ}}=\frac{5}{2}\left(\frac{y_{1}}{y_{0}} \frac{y_{2}}{y_{0}}-\frac{y_{3}}{y_{0}}\right)$.

We explain a little about the A-variation of polarized Hodge structure of the quintic $V . \mathcal{H}^{V}=\left(\mathcal{H}_{\mathcal{O}}^{V}, \nabla^{\text {middle }}, \mathcal{H}_{\mathbf{Z}}^{V}, S, \mathcal{F}\right)$, constructed in 3.6, is indeed a polarized variation of Hodge structure on the punctured disc. The idea of proof is to verify the following two properties.

(1) Let $\nabla=\nabla^{\text {middle }}$. Then $\nabla \mathcal{F}^{p} \subset \Omega_{\mathcal{K} \mathcal{M}(V)}^{1} \otimes \mathcal{F}^{p-1}$ for all $p$.

(2) $\mathcal{F}^{p} \oplus \overline{\mathcal{F}^{3-p+1}}=\mathcal{H}_{\mathcal{O}}$ for all $p$.

(1) is easy, because the small quantum product $*_{\text {small }}$, from which A-model connection actually defined, is compatible with Hodge structure (see, e.g., [CoK99, 8.5.4]). (2) is hard. One uses the results of [CaKS87] and [CaKS86] (see, e.g., [CoK99, 8.5.4]).

\subsection{Proof of (4) in Introduction}

Proof. We prove the equivalence of (3) and (4) in Introduction.

We show $0(3) \Rightarrow 0(4)$. Let $S^{*}:=\mathcal{K} \mathcal{M}(V)$ and $S:=\overline{\mathcal{K}} \mathcal{M}(V)$ for A-model, and $S^{*}:=\mathcal{M}\left(V^{\circ}\right)$ and $S:=\overline{\mathcal{M}}\left(V^{\circ}\right)$ for B-model. $S$ is endowed with the log structure associated to the divisor $S \backslash S^{*}$. In both cases, the variation of polarized Hodge structure on $S^{*}$ extends uniquely to a log variation of polarized Hodge structure on $S$ by the log Hodge theoretic interpretation [KU09, 2.5.14] of nilpotent orbit theorem of Schmid [S73]. The specified sections $1=T_{0}$ for A-model and $\tilde{\Omega}=e_{0}$ for B-model extend over $S$ as the canonical extensions as mentioned above, and the specified sections $[\mathrm{pt}]=T^{0}$ for A-model and $g_{0}=e^{0}(3.5,3.6)$ for B-model extend over $S$ as invariant sections.

The converse implication $0(4) \Rightarrow 0(3)$ is obvious.

Another Direct Proof. If we use the correspondence table in 3.6, the implication 0 $(3) \Rightarrow 0$ (4) is verified directly as follows.

Let $S^{*}$ and $S$ be as in the above Proof. We use $u$ and $q$ from 3.4 for $A$-model and 3.3 for $B$-model, respectively. Let $\tilde{S}^{*}:=\mathbf{R} \times i(0, \infty)$ and $\tilde{S}^{\log }:=\mathbf{R} \times i(0, \infty]$ be the universal covering of $S^{*}$ and $S^{\log }$, respectively. The coordinate $u$ of $\tilde{S}^{*}$ extends over $\tilde{S}^{\log }$. Denote $0+i \infty \in \tilde{S}^{\log }$ by $u_{0}$ and its image $\overline{0}+i \infty \in S^{\log }$ by $b$. These points $u_{0}$ and $b$ lie over the point $q=0 \in S$ which corresponds to $q_{0}$ for $A$-model and $p_{0}$ for $B$-model, respectively.

Let $H_{\mathbf{Z}}:=\mathcal{H}_{\mathbf{Z}}^{V}$ for $A$-model, and $H_{\mathbf{Z}}:=\mathcal{H}_{\mathbf{Z}} V^{\circ}$ for $B$-model. The local system $H_{\mathbf{Z}}$ over $S^{*}$, endowed with the respective symplectic basis $s_{0}, s_{1},-s^{0}, s^{1}$ from (4A) and (4B) in 3.6, extends as a local system over $S^{\log }$ with the extended symplectic basis (cf. [KU09, 2.3.5], [O03], [U01]). Note that to fix a base point $u=u_{0}$ on $\tilde{S}^{\log }$ is equivalent to fix a base point $b$ on $S^{\log }$ and also a branch of $(2 \pi i)^{-1} \log q$. Regarding the stalk $H_{0}:=H_{\mathbf{Z}, u_{0}}=H_{\mathbf{Z}, b}$ as a constant sheaf on $S^{\log }$, we have an isomorphism $\mathcal{O}_{S}^{\log } \otimes H_{\mathbf{Z}} \simeq$ $\mathcal{O}_{S}^{\log } \otimes H_{0}$ of $\mathcal{O}_{S}^{\log }$-modules whose restriction induces the identity $1 \otimes H_{\mathbf{Z}, b}=1 \otimes H_{0}$ ([KU09, 2.3.2], see Appendix to Section 1).

The direct image $\tau_{*}\left(\mathcal{O}_{S}^{\log } \otimes H_{\mathbf{Z}}\right)$, via $\tau: S^{\log } \rightarrow S$ from 1.2 , yields the canonical extension $\mathcal{O}_{S}$-module of Deligne over $S$ of $H_{\mathcal{O}}$ over $S^{*}$. The bases $T_{0}, T_{1}, T^{1}, T^{0}$ from 
$(2 \mathrm{~A})$ and $e_{0}, e_{1}, e^{1}, e^{0}$ from $(2 \mathrm{~B})$ in 3.6 yield monodromy invariant bases of $\mathcal{O}_{S}$-modules respecting the Hodge filtration for each case. These bases extend over $q=0$ and yield the canonical extensions of the respective Hodge filtrations. This is because, by $(6 \mathrm{~A})$ and $(6 \mathrm{~B})$ in $3.6, T_{j}, T^{j}$ and $e_{j}, e^{j}$ are linear combinations of respective $s_{j}, s^{j}$ with coefficients in $\mathcal{O}_{S, b}^{\log }=\mathbf{C}\{q\}[u]$ ( $u$ is considered as a multi-values function on a neighborhood of $q=0$ in $\left.S^{*}\right)$, and $s_{j}, s^{j}$ are $\mathcal{O}_{S, b}^{\log }$-linear combination of the elements

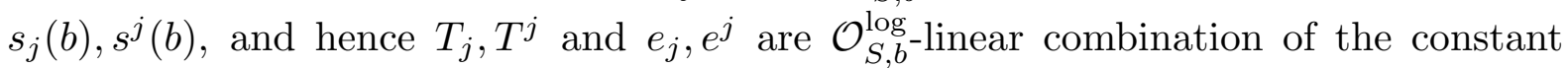
sections $s_{j}(b), s^{j}(b) \in H_{\mathbf{Z}, b}=H_{0}$. For $h \in \mathcal{O}_{S, b}^{\log }$ appeared as the last coefficients, $h$ is monodromy invariant holomorphic function on $S^{*}$ which satisfies $\lim _{q \rightarrow 0} q h=0$, and hence $q=0$ is a removable singularity of $h$. Thus the value of $h$ at $q=0$ is determined. (Using the table in 3.6, we can also examine the above argument by a direct computation.)

Now we verify that the polarized variation of Hodge structure $H$ of type $(\Lambda, \Gamma)$ over $S^{*}$ extends uniquely to a log variation of polarized Hodge structure of type $\Phi=(\Lambda, \Sigma, \Gamma)$ over $S$, where $\Gamma=\exp (\mathbf{Z} N)$ and $\Sigma=\{\{0\}, \sigma\}$ with $N$ and $\sigma$ from 0 (4). (Note that $N$ here is $-N$ of $N$ in Sections 1-2.) In fact, we have already seen the canonical extensions of Hodge filters as $\mathcal{O}_{S}$-modules. Admissibility $1.3(1)$ is obvious in pure case. Big Griffiths transversality 1.3 (7) follows from the above choice of the bases $T_{0}, T_{1}, T^{1}, T^{0}$ and $e_{0}, e_{1}, e^{1}, e^{0}$. Positivity $1.3(3)$ is verified by direct computation using these bases and the polarizations $S$ from (1A) and $Q$ from (1B) in 3.6 in the following way. We do this for B-model case. A-model case is analogous. For $u=u_{0}$, let $F(0) \in \check{D}$ be the fixed Hodge filtration corresponding to the basis $e_{0}\left(u_{0}\right), e_{1}\left(u_{0}\right), e^{1}\left(u_{0}\right), e^{0}\left(u_{0}\right)$. Let $F_{y}:=$ $\exp (i y(-N)) F(0) \in \check{D}$. Then, $v_{3}(y):=\exp (i y(-N)) e_{0}\left(u_{0}\right)$ and $\exp (i y(-N)) e_{1}\left(u_{0}\right)$ form a basis of $F_{y}^{2}$ respecting $F_{y}^{3}$. From this, we can compute a basis $v_{2}(y)$ of $F_{y}^{2} \cap \overline{F_{y}^{1}}=$ $F_{y}^{2} \cap\left(\overline{F_{y}^{3}}\right)^{\perp}$, where ${ }^{\perp}$ is for $Q$. We can check that the coefficients of the highest terms in the variable $y$ of the Hodge norms $i^{3} Q\left(v_{3}(y), \overline{v_{3}(y)}\right)$ and $i Q\left(v_{2}(y), \overline{v_{2}(y)}\right)$ are both positive.

The extension of the specific sections has already seen.

\subsection{Comments}

(i) We want to see the relation of $N_{\beta}$ from 2.3 and the monodromy $\operatorname{logarithm} N$ from 0 (4). It is enough to see the relation of the following $A$ and $B$.

$$
A:=\left(\begin{array}{cccc}
0 & 0 & 0 & 0 \\
1 & 0 & 0 & 0 \\
0 & 1 & 0 & 0 \\
0 & 0 & 1 & 0
\end{array}\right), \quad B:=\left(\begin{array}{cccc}
0 & 0 & 0 & 0 \\
1 & 0 & 0 & 0 \\
0 & 5 & 0 & 0 \\
0 & 0 & 1 & 0
\end{array}\right)
$$

We want to find $g \in \operatorname{Sp}(4, \mathbf{Q})$ and a non-zero real number $r$ such that $B=r^{-1} g A g^{-1}$. Such $g$ and $r$ are given by $g=\left(\begin{array}{cccc}a & 0 & 0 & 0 \\ b & r a & 0 & 0 \\ e & 5 r b & 5 r^{2} a & 0 \\ h & r e & 5 r^{2} b & 5 r^{3} a\end{array}\right)$ such that $2 a e=5 b^{2}$ and 
$5 r^{3} a^{2}=1$. For example, for $a=5, b=e=2$ and $r=1 / 5, g=\left(\begin{array}{cccc}5 & 0 & 0 & 0 \\ 2 & 1 & 0 & 0 \\ 2 & 2 & 1 & 0 \\ h & 2 / 5 & 2 / 5 & 1 / 5\end{array}\right)$.

We cannot find $g$ in $\operatorname{Sp}(4, \mathbf{Z})$, because of the second condition for $g$.

(ii) For general Calabi-Yau threefolds satisfying the Integrality Conjecture, we can show the equivalence of the formulation (4) and formulations (1)-(3) in Introduction. The result will appear somewhere.

(iii) Open mirror symmetry started by Walcher [W07]. [MW09], [PSW08], [LiLY12] etc. are related works. After [MW09], in B-model side, a main subject is a normal function associated with a family of quintic-mirrors and specific algebraic cycle homologous to 0 on it, especially its values for the normalized holomorphic 3 -forms, which is called domainwall tension in physics. This normal function extends over the Néron model from Corollary in 2.4. This extended normal function is an interesting topic to study.

\section{REFERENCES}

[CaK82] E. Cattani and A. Kaplan, Polarized mixed Hodge structures and the local monodromy of a variation of Hodge structure, Invent. Math. 67 (1982), 101-115.

[CaKS86] E. Cattani, A. Kaplan, and W. Schmid, Degeneration of Hodge structures, Ann. Math. 123 (1986), 457-535.

[CaKS87] _ $L^{2}$ and intersection cohomologies for a polarized variation of Hodge structure, Invent. Math. 87 (1987), 217-252.

[CoK99] D. A. Cox and S. Katz, Mirror symmetry and algebraic geometry, Math. Surveys and Monographs, vol. 68, AMS, 1999, pp. 469.

[CDGP91] P. Candelas, C. de la Ossa, P. S. Green, and L. Parks, A pair of Calabi-Yau manifolds as an exactly soluble superconformal theory, Phys. Lett. B 258 (1991), 21-74.

[D70] P. Deligne, Équations differentielles à points singuliers réguliers, Lect. Notes in Math. No. 163, Springer-Verlag, 1970.

[D97] P. Deligne, Local behavior of Hodge structures at infinity, in Mirror Symmetry II (B. Greene and S.-T. Yau, eds.), AMS/IP Stud. Adv. Math. 1, 1997, 683-699.

[Gi96] A. Givental, Equivariant Gromov-Witten invariants, Internat. Math. Res. Notices (1996), 613-663.

[Gi97p] _ A mirror theorem for toric complete intersections, preprint (1997), AG/9701016.

[Gr68a] P. A. Griffiths, Periods of integrals on algebraic manifolds, I. Construction and properties of the modular varieties, Amer. J. Math. 90 (1968), 568-626.

[Gr68b] Periods of integrals on algebraic manifolds, II. Local study of the period mapping, Amer. J. Math. 90 (1968), 805-865.

[Gr69] _ On the periods of certain rational integrals, I, II., Ann. of Math. 90 (1969), 460495, 498-541.

[K86] M. Kashiwara, A study of variation of mixed Hodge structure, Publ. R.I.M.S., Kyoto Univ. 22 (1986), 991-1024.

[KN99] K. Kato and C. Nakayama, Log Betti cohomology, log étale cohomology, and log deRham cohomology of log schemes over C, Kodai Math. J. 22 (1999), 161-186.

[KNU08] K. Kato, C. Nakayama and S. Usui, SL(2)-orbit theorem for degeneration of mixed Hodge structure, J. Algebraic Geometry 17 (2008), 401-479.

[KNU09] _ Classifying spaces of degenerating mixed Hodge structures, I: Borel-Serre spaces, Advanced Studies in Pure Math. 54: Algebraic Analysis and Around, 2009, 187-222. 
[KNU11] _ Classifying spaces of degenerating mixed Hodge structures, II: Spaces of SL(2)orbits, to appear in Kyoto J. Math. 51-1: Nagata Memorial Issue (2011), 149-261. (available in $\operatorname{arXiv}$ ).

[KNU12] _ Classifying spaces of degenerating mixed Hodge structures, III: Spaces of nilpotent orbits, to appear in J. of Alg. Geom. (accepted 2012), old version arXiv:1011.4353.

[KO68] N. Katz and T. Oda, On the differentiation of De Rham cohomology classes with respect to parameters, J. Math. Kyoto Univ. 8 (1968), 199-213.

[KU99] K. Kato and S. Usui, Logarithmic Hodge structures and classifying spaces (summary), in CRM Proc. \& Lect. Notes: The Arithmetic and Geometry of Algebraic Cycles, (NATO Advanced Study Institute / CRM Summer School 1998: Banff, Canada) 24 (1999), 115130.

[KU02] _ Borel-Serre spaces and spaces of SL(2)-orbits, Advanced Studies in Pure Math. 36: Algebraic Geometry 2000, Azumino, (2002), 321-382.

[KU09] _ Classifying spaces of degenerating polarized Hodge structures, Ann. Math. Studies, Princeton Univ. Press, vol. 169, Princeton, 2009, pp. 288.

[LiLY12] S. Li, B. Lian, and S.-T. Yau, Picard-Fuchs equations for relative periods and Abel-Jacobi map for Clabi-Yau hypersurfaces, Amer. J. Math. 134-5 (2012), 1345-1384.

[LLuY97] B. Lian, K. Liu, and S.-T. Yau, Mirror principle I, Asian J. Math. 1 (1997), 729-763.

[M93] D. Morrison, Mirror symmetry and rational curves on quintic threefolds: A guide for mathematicians, J. of AMS 6-1 (1993), 223-247.

[M97] _ Mathematical aspects of mirror symmetry, in Complex algebraic geometry (Park City, UT, 1993), IAS/Park City Math. Ser. 3, AMS (1997), 265-327.

[MW09] D. Morrison and J. Walcher, D-branes and normal functions, Adv. Theor. Math. Phys. 13-2 (2009), 553-598.

[O03] A. Ogus, On the logarithmic Riemann-Hilbert correspondences, Documenta Math. extra volume: Kazuya Kato's fiftieth birthday (2003), 655-724.

[P98] R. Pandharipande, Rational curves on hypersurfaces [after A. Givental], Séminaire Bourbaki 848 (1997-98), AG/9806113.

[PSW08] R. Pandharipande, J. Solomon, and J. Walcher, Disk enumeration on the quintic 3-fold, J. Amer. Math. Soc. 21-4 (2008), 1169-1209.

[S73] W. Schmid, Variation of Hodge structure: The singularities of the period mapping, Invent. Math. 22 (1973), 211-319.

[U84] S. Usui, Variation of mixed Hodge structure arising from family of logarithmic deformations II: Classifying space, Duke Math. J. 51-4 (1984), 851-875.

[U01] _ Recovery of vanishing cycles by log geometry, Tohoku Math. J. 53-1 (2001), 1-36.

[U08] _ Generic Torelli theorem for quintic-mirror family, Proc. Japan Acad. 84, Ser. A, No. 8 (2008), 143-146.

[W07] J. Walcher, Opening mirror symmetry on the quintic, Commun. Math. Phys. 276 (2007), $671-689$.

Sampei USUI

Graduate School of Science

Osaka University

Toyonaka, Osaka, 560-0043, Japan

usui@math.sci.osaka-u.ac.jp 